\title{
Some Formal Results on Positivity, Stability, and Endemic Steady-State Attainability Based on Linear Algebraic Tools for a Class of Epidemic Models with Eventual Incommensurate Delays
}

\author{
M. De la Sen $\left(\mathbb{D},{ }^{1}\right.$ R. Nistal, ${ }^{1}$ S. Alonso-Quesada ${ }^{(D)},{ }^{1}$ and A. Ibeas $\mathbb{D}^{2}$ \\ ${ }^{1}$ Institute of Research and Development of Processes IIDP, University of the Basque Country, Campus of Leioa, Leioa (Bizkaia), \\ P.O. Box 48940, Spain \\ ${ }^{2}$ Department of Telecommunications and Systems Engineering, Universitat Autònoma de Barcelona, UAB, 08193-Barcelona, Spain \\ Correspondence should be addressed to M. De la Sen; manuel.delasen@ehu.eus
}

Received 20 December 2018; Revised 20 May 2019; Accepted 16 June 2019; Published 8 July 2019

Academic Editor: Cengiz Çinar

Copyright (C) 2019 M. De la Sen et al. This is an open access article distributed under the Creative Commons Attribution License, which permits unrestricted use, distribution, and reproduction in any medium, provided the original work is properly cited.

\begin{abstract}
A formal description of typical compartmental epidemic models obtained is presented by splitting the state into an infective substate, or infective compartment, and a noninfective substate, or noninfective compartment. A general formal study to obtain the reproduction number and discuss the positivity and stability properties of equilibrium points is proposed and formally discussed. Such a study unifies previous related research and it is based on linear algebraic tools to investigate the positivity and the stability of the linearized dynamics around the disease-free and endemic equilibrium points. To this end, the complete state vector is split into the dynamically coupled infective and noninfective compartments each one containing the corresponding state components. The study is then extended to the case of commensurate internal delays when all the delays are integer multiples of a base delay. Two auxiliary delay-free systems are defined related to the linearization processes around the equilibrium points which correspond to the zero delay, i.e., delay-free, and infinity delay cases. Those auxiliary systems are used to formulate stability and positivity properties independently of the delay sizes. Some examples are discussed to the light of the developed formal study.
\end{abstract}

\section{Introduction}

Epidemic models have been widely studied in the last decades involving several inter-actuating subpopulations with mutual coupled dynamics. Important properties which have to be required to the epidemic models for their well-posedness are their solution positivity under any given nonnegative initial conditions as well as their stability conditions around one of the equilibrium points. Thus, relevant background literature has dealt with the study of these issues for different types of epidemic models, in both continuous and discrete-time [1-5]. A general result is that the disease-free equilibrium point is locally asymptotically stable when the endemic one is not reachable and if this last one is reachable (i.e., allocated within the closed first orthant of the real space including any trajectory solution) then it is locally stable while the diseasefree equilibrium one is unstable. The reachability of the endemic equilibrium point, as well as the stability properties of the equilibrium points, is definitely linked with the value of the so-called reproduction number (sometimes referred to as "basic reproduction number" or "basic reproduction ratio" in the literature $[4,5]$ ). If such a reproduction number is less than unity then the disease-free equilibrium point is locally asymptotically stable and the endemic one is unattainable (or unreachable) since it has some negative component for some infective subpopulation. This fact implies the global asymptotic stability to the disease-free equilibrium point provided that all the infective subpopulations are asymptotically stable. However, if the reproduction number exceeds unity, then the disease-free equilibrium point is unstable and the endemic equilibrium point is reachable (or attainable) so that the disease becomes permanent through time. The frontier between both situations typically occurs when the reproduction number is unity. See, for instance, [6-12] and 
some references therein. In those studies, the first orthant of the space state is an invariant subspace as a result of the nonnegativity properties of the state trajectory solutions which is also an invoked property of other biological problems related to species evolution dynamics. See, for instance, [13] and some references therein. Since the reproduction number is a very important tool to have a biological insight about if the disease is either permanent (more than one contagion per infectious individual, in average) or it extinguishes (less than one contagion per infectious individual, in average) many efforts have been done to calculate it for different epidemic models, $[4,5]$. The calculation is usually performed in a case-by-case fashion providing the value of the reproduction number for each particular considered model. It turns out that there are no general analysis tools available in the background literature to discuss those properties based on general reasonable and generic assumptions independent of the particular epidemic model. Although these kinds of results are known from the background literature in a variety of epidemic models, they are revisited and presented here in a general framework as a fruitful combination of algebraic results based on positivity and stability of the linearized systems around the equilibrium points. Thus, a general technique to obtain the reproduction number and discuss the stability properties of equilibrium points for any type of compartmental epidemic models is proposed and discussed. The formal study is based on linear algebraic tools to investigate the positivity and the stability of the linearized dynamics around the disease-free and endemic equilibrium points. Consequently, our results unify previous research in this respect. To this end, the original state vector is split into the dynamically coupled infective and noninfective compartments each one containing the corresponding state components. The infective compartment includes the dynamics of all the infective subpopulations in the model, typically, the exposed, asymptomatic and symptomatic infectious and dead-infective, if considered in the model. The noninfective compartment is composed of the subpopulations being free of the disease and typically contains the dynamics of the susceptible and the recovered. For the subsequent study development, the characteristics of the state transition matrices of the linearized infective compartments around the equilibrium points of the epidemic models, which are Metzler matrices, [14, 15], as well as their stability properties and the relations of their properties to those of their opposite $M$ - matrices, are seen to be crucial mathematical tools. The main reason is that the linearized epidemic system versions around the equilibrium points have also to possess nonnegative solution trajectories since the whole epidemic model has nonnegative solution trajectories under any given nonnegative initial conditions. In particular, such state transition matrices define the so-called next generation matrix [4] of the infective compartment. The maximum modulus of such a matrix is the relevant parameter to characterize the stability of the infective compartment and it often determines the disease reproduction number. It is also a crucial fact in the analysis the property that the transmissions matrices $F$ of such linearized systems around the equilibrium points are nonnegative. If the Perron root of the auxiliary matrix (next generation matrix) $F V^{-1}$, with
$(-V)$ being the disease transition matrix, i.e., its maximum real positive eigenvalue, $[2,3,16]$, is less than unity then the linearized system around the disease-free equilibrium point is proved to be locally asymptotically stable and conversely. It is also proved that the reproduction number is linked to the Perron root of the above auxiliary matrix, which coincides with its spectral radius in typical examples of epidemic models. The presence of delays is an important modeling tool in epidemiology $[11,17]$ in cases when there are successive outbreaks and regrowths of the disease intensity caused by increase of the transmission vector numbers or external immigration to the environment under study in the model. Therefore, once the above general algebraic framework is set for delay-free models, the above study is extended to epidemic models under, in general, incommensurate (in the sense that they are not all integer multiple of the smaller, or base, delay) state point delays, [18]. The case of commensurate delays is a particular case of the above one when all the delays are integer multiples of the base delay [19-23]. Two auxiliary delay-free systems are defined related to the linearization processes around the equilibrium points which correspond to the zero delay, i.e., delay-free, and infinity delay cases. Those auxiliary systems are used to formulate stability and positivity properties, independent of the delay sizes, for the whole epidemic model. As a result of the developed formal treatment, the reproduction number in the delayed case is not directly given by the spectral radii of certain matrices related to the delay-auxiliary free and infinity delay linearized systems around the equilibrium points. Those spectral radii are, in general, lower bounds of the reproduction number. In particular, they give a guaranteed worst-case measure of guaranteed stability of the disease-free equilibrium point.

The remaining main body is organized in two more sections referred to the model analysis in the delay-free case and under the presence of delays. The positivity and stability of the solutions in both cases are formally discussed in a general context, rather than for specific models, based on the linearization analysis on the infective compartment for the disease-free and endemic equilibrium points. Illustrative examples referred to the formal links of the presented mathematical framework to particular epidemic models are discussed. Two given appendices are given which contain auxiliary technical results on stability of Metzler and $M$ matrices and on the uniqueness of the equilibrium points.

\subsection{Notation}

$$
\begin{aligned}
& \bar{p}=\{1,2, \ldots, p\}, \\
& \mathbf{R}_{0+}=\mathbf{R}_{+} \cup\{0\}=\{r \in \mathbf{R}: r \geq 0\} ; \mathbf{R}_{+}=\{r \in \mathbf{R}: r>0\} \\
& \mathbf{R}_{0-}=\mathbf{R}_{-} \cup\{0\}=\{r \in \mathbf{R}: r \leq 0\} ; \mathbf{R}_{-}=\{r \in \mathbf{R}: r<0\} \\
& \mathbf{C}_{0+}=\mathbf{C}_{+} \cup\{\mathbf{i} \omega: \omega \in \mathbf{R}\}=\{z \in \mathbf{C}: \operatorname{Re} z \geq 0\} \\
& \mathbf{C}_{+}=\{z \in \mathbf{C}: \operatorname{Re} z>0\} ; \mathbf{i}=\sqrt{-1} \text { is the complex } \\
& \text { unity; } C(0,1) \text { is the closed circle in } \mathbf{C} \text { centred at } 0 \\
& \text { and radius one of boundary } \partial C(0,1) \text { (that is, the unit } \\
& \text { circumference), } \\
& \mathbf{C}_{0-}=\mathbf{C}_{-} \cup\{\mathbf{i} \omega: \omega \in \mathbf{R}\}=\{z \in \mathbf{C}: \operatorname{Re} z \leq 0\} \\
& \mathbf{C}_{-}=\{z \in \mathbf{C}: \operatorname{Re} z<0\} ;
\end{aligned}
$$


If $A=\left(A_{i j}\right) \in \mathbf{R}^{p \times q}$ then (1) $A \geq 0$ (in words: $A$ is a nonnegative matrix) means that $A_{i j} \geq 0 ; \forall i \in \bar{p}, \forall j \in \bar{q}$. An equivalent notation is $A \in \mathbf{R}_{0+}^{p \times q}$. Similarly, if $A, B \in \mathbf{R}^{p \times q}$ then $A \succeq B$ means that $A-B \succeq 0$ and $A \preceq 0$ means that $-(A) \succeq 0$.

(2) $A>0$ (in words: $A$ is a positive matrix) means that $A(\neq 0) \geq 0$, that is, $A \geq 0$ with at least one positive entry. Similarly, if $A, B \in \mathbf{R}^{p \times q}$ then $A>B$ means that $A-B>0$ and $A \prec 0$ means that $-(A)>0$.

(3) $A \gg 0$ (in words: $A$ is a strictly positive matrix) means that $A_{i j}>0 ; \forall i \in \bar{p}, \forall j \in \bar{q}$. An equivalent notation is $A \in \mathbf{R}_{+}^{p \times q}$. Similarly, if $A, B \in \mathbf{R}^{p \times q}$ then $A \succ>B$ means that $A-B \succ \succ 0$ and $A \prec \prec 0$ means that $-(A) \succ \succ 0$,

It turns out that $A \succ>0 \Longrightarrow A \succ 0 \Longrightarrow A \succeq 0$.

The logic conjunction ("And") and logic disjunction ("Or") of propositions are defined by the symbols " $\wedge$ " and "V," respectively. The logic negation ("No") is defined by the symbol “ $\neg$ ". For instance, if $A \in \mathbf{R}^{p \times q}$ then $\neg A=\left(A_{i j}\right)>0$ means that $A_{i j}<0$ for some $(i, j) \in \bar{p} \times \bar{q}$.

For vectors $v \in \mathbf{R}^{n}$, similar nonnegativity, positivity, and strictly positivity notations are used, namely, $v \geq 0, v>0$, $v>>0$ and the corresponding nonpositivity, negativity, and strict negativity counterpart notations.

$I_{n}$ is the identity $n$-matrix, and superscript $T$ denotes a matrix transposition,

$\rho(A)=\left|\lambda_{\text {max }}(A)\right|$ is the spectral radius of $A \in \mathbf{R}^{p \times p}$,

$A \in \mathbf{R}^{p \times p}$ is a Metzler matrix, denoted by $A \in M_{E}^{p \times p}$, if $A_{i j} \geq 0 ; \forall i, j(\neq i) \in \bar{p}$,

$A \in \mathbf{R}^{p \times p}$ is an $M$-matrix if $A_{i i} \geq 0$ and $A_{i j} \leq 0 ; \forall i, j(\neq$ i) $\in \bar{p}$,

$\|F(s)\|_{\infty}$ is the $H_{\infty}$ - norm of complex rational matrices $F: \mathbf{C} \longrightarrow C^{p \times q}$ which are analytic in $\operatorname{Re} s>0 ; s \in \mathbf{C}$. The argument "s" stands for the Laplace transform argument.

\section{Results for Delay-Free Epidemic Models}

A general compartmental disease model of dimension $(m+$ $n$ ) can be written as [1]

$$
\begin{aligned}
\dot{x}(t) & =F(x(t), y(t))-V(x(t), y(t)) \\
& =(F-V) x(t)-f(x(t), y(t)) \\
\dot{y}(t) & =g(x(t), y(t))
\end{aligned}
$$

for any given $z(0)=\left(x^{T}(0), y(0)^{T}\right)^{T}$, where $n$ and $m$ are the respective dimensions of the disease and nondisease compartments of respective state vectors $x(t)$ and $y(t)$, $F \in \mathbf{R}^{n \times n}$ and $(-V) \in \mathbf{R}^{n \times n}$ are, respectively, the disease transmission and state transition matrices of the linearized system around the disease-free equilibrium point, and

$$
\begin{aligned}
f(x(t), y(t))= & (F-V) x(t)-F(x(t), y(t)) \\
& +V(x(t), y(t))=\theta(z(t)) G(z(t))
\end{aligned}
$$

describes the higher-order contributions to the infective compartment dynamics, where $z(t)=\left(x^{T}(t), y^{T}(t)\right)^{T}$ is the whole model state vector, $\theta(z(t))$ is the scalar positive bounded incidence rate, and $G(z(t))=F(z(t)) z(t)$, where $G(z(t)) \in \mathbf{R}^{n \times(n+m)}$ is a partitioned block matrix function of the form $G(z(t))=\left[G_{x x}(y(t)) G_{x y}(y(t))\right]$. We can also rename, by obvious reasons, the state vectors $x(t)$ and $y(t)$ of the disease and nondisease compartments as the infective and noninfective compartment (or substates) of the epidemic model, respectively. The following assumptions are made.

Assumptions 1. $F_{i}(0, y(t))=V_{i}(0, y(t))=0 ; \forall i \in \bar{n}=$ $\{1,2, \ldots, n\}, F_{i}(x(t), y(t)) \geq 0, V_{i}(x(t), y(t)) \leq 0$ when $x_{i}=0$, and $\sum_{i=1}^{n} V_{i}(x(t), y(t)) \geq 0$ for all $x(t), y(t) \geq 0$.

The conditions of Assumptions 1 are invoked for the nonnegativity of the state trajectory solution for initial conditions in the first orthant and for the local stability of the disease-free equilibrium point in the absence of infection, i.e., under a zero reproduction number. See [1-3] for more specific details. In [1-3], the matrices $F$ and $V$ are real square $n$-matrices defined as the following Jacobian matrices around the disease-free equilibrium point $z_{d f}=\left(0^{T}, y_{d f}^{T}\right)^{T}$ :

$$
\begin{aligned}
& F=\left[\frac{\partial F_{i}\left(0, y_{d f}\right)}{\partial x_{j}}\right] ; \\
& V=\left[\frac{\partial V_{i}\left(0, y_{d f}\right)}{\partial x_{j}}\right]
\end{aligned}
$$

The following assumptions on the above Jacobian matrices are reasonable in the context of epidemic models [1]. The notations $F, V$ stand through the manuscript for the Jacobian matrices around the disease-free equilibrium point. The notations $F_{e}, V_{e}$ stand for their counterparts around the endemic equilibrium point $x_{\text {end }}$ while the notations $F_{0}, V_{0}$ stand for any generic equilibrium point $z_{0}$. It is well-known in many typical existing epidemic models that $z_{\text {end }}$ and $z_{d f}$ are coincident when the reproduction number is unity.

Assumptions 2. (a) The disease-free equilibrium point $z_{d f}=$ $\left(0^{T}, y_{d f}^{T}\right)^{T}$ is unique and the noninfective substate dynamics $\dot{y}(t)=g(0, y(t))$ is locally asymptotically stable.

(b) The minus transition matrix $V$ of the infective states of the linearized infective subsystem around the disease-free equilibrium point is a nonsingular $M$-matrix with $V^{-1}>0$ while the corresponding transmission matrix fulfills $F \geq 0$.

The above assumptions are very relevant from a physical point of view having in mind that $(-V)$ and $F$ are, respectively, the transition and transmission matrices of the linearized system around the disease-free equilibrium point while ( $F$ $V)$ is the matrix of the dynamics of such a system. It turns out, in practice, that $F=0$ if and only if the coefficient transmission rate $\beta$ is zero, that is, there is no disease contagion in practice. Some conditions for the uniqueness of the disease-free equilibrium point are given in Theorem B.1 of Appendix B based on the implicit function theorem. Some conditions are also given for the uniqueness of the endemic equilibrium point in Theorem B.2 of Appendix B based on the Rouché-Frobenius test for compatibility of solutions in algebraic systems. This alternative way of proof is taken on the basis that distinct endemic equilibrium points can have 
distinct disease and nondisease endemic steady-state equilibrium compartments while the disease-free equilibrium has always zero infective substates. Both theorems are also valid for the case of presence of internal delays. Note that Assumption 2(b) can be stated in the following equivalent form (see Theorem A.1 of Appendix A):

Assumption 3(b). The transition matrix $(-V)$ of the infective state of the linearized system around the disease-free equilibrium point is a stability Metzler matrix while the corresponding transmission matrix fulfills $F \geq 0$.

Note that Assumption 3(b) is of a clear interpretation for the linearization around the disease-free equilibrium point while its equivalent form of Assumption 2 is easy to test since it is not needed to calculate the allocation of the eigenvalues of the matrix $(-V)$. Note also that $F=F\left(R_{0}, y_{d f}\right) \geq 0$ depends on the disease-free equilibrium substate of the noninfective components and on the reproduction number $R_{0}$ associated with the disease propagation. If $R_{0}=0$ then $F=F\left(0, y_{d f}\right)=$ 0 . Also, it usually occurs that if the epidemic model has only a single constant transmission coefficient rate $\beta$, then $R_{0}=0$ and $F=0$ if $\beta=0$. This section gives some elementary results on positivity of the solutions and stability for the linearized system around the disease-free equilibrium point in the case of absence of modelling delays. The results are relevant since if the linearized system around the disease- free equilibrium point fails to be either positive or, respectively, stable then the whole system cannot be either positive or, respectively, stable. Two basic auxiliary results to be invoked concerning certain technical relations between $\mathrm{M}$-matrices and Metzler matrices and their stability properties are given in Appendix A. The following result on stability and positivity of the linearized infective substate is derived under some of the conditions of Assumptions 1-2:

\section{Theorem 1. The following properties hold.}

(i) The linearization of the epidemic model (1)-(2) around the disease-free equilibrium point has a nonnegative infective substate trajectory solution for any initial conditions $x(0) \succeq 0$ and $y(0) \succeq 0$ (i.e., the infective substate is nonnegative for all time) if and only if $(F-V) \in M_{E}^{n \times n}$. A sufficient condition is that $(-V) \in M_{E}^{n \times n}$ and $F \geq 0$.

(ii) A necessary condition for an epidemic model (1)-(2) to have a nonnegative solution trajectory, irrespective of the given particular initial conditions $x(0) \geq 0, y(0) \geq 0$, is that $(F-$ $V) \in M_{E}^{n \times n}$.

(iii) The linearized infective substate around the diseasefree equilibrium point has a nonnegative solution which is uniformly bounded for all time with $x_{L}(t) \geq 0 ; \forall t \in \mathbf{R}_{0+}$ and $x_{L}(t) \longrightarrow 0$ exponentially as $t \longrightarrow \infty$ for any given initial conditions $x_{L}(0)=x(0) \geq 0, y_{L}(0)=y(0) \geq 0$ if and only if any of the two equivalent conditions given below holds:

(1) $(F-V) \in M_{E}^{n \times n}$ is a stability matrix.

(2) $(F-V) \in M_{E}^{n \times n}$ is nonsingular (or, equivalently, $(V-F)$ is a nonsingular M-matrix) and $(V-F)^{-1}>0$.

$A$ joint sufficiency-type condition for any of the above Conditions (1)-(2) to hold is that $(-V) \in M_{E}^{n \times n}$ is nonsingular (or, equivalently, $V$ is a nonsingular $M$-matrix), $V^{-1}>0, F \geq 0$ and $\rho\left(F V^{-1}\right)<1$. (iv) The linearized infective substate around the disease-free equilibrium point is nonnegative for any given initial conditions $x_{L}(0)=x(0) \geq 0, y_{L}(0)=y(0) \geq 0$ and unstable if some of the conditions given below hold:

(1) $(F-V) \in M_{E}^{n \times n}$ is not a stability matrix.

(2) $(F-V) \in M_{E}^{n \times n}$ and $(V-F)^{-1}$ either does not exist or if it exists is not positive.

(3) $(-V) \in M_{E}^{n \times n}$ and it exists as $V^{-1}$ which is not positive, $F \geq 0$ and $\rho\left(F V^{-1}\right)>1$.

Proof. The solution of the linearized infective substate around the disease-free equilibrium point is

$$
\begin{aligned}
x_{L}(t) & =e^{(F-V) t} x_{L}(0) \\
& =e^{-V t}\left(x_{L}(0)+\int_{0}^{t} e^{V \tau} F x_{L}(\tau) d \tau\right)
\end{aligned}
$$

It turns out that, for any $x_{L}(0) \geq 0, x_{L}(t) \geq 0$ since $e^{(F-V)(t-\tau)}>0$ for any $\tau(\leq t), t \in \mathbf{R}_{0+}$ and $(F-V) \in M_{E}^{n \times n}$ [14], then, $x_{L}(t) \geq 0 ; \forall t \in \mathbf{R}_{0+}$ for any given $x_{L}(0) \geq 0$ if $(F-V) \in M_{E}^{n \times n}$ (first term of the solution $\left.x_{L}(t)\right)$ or if $(-V) \in M_{E}^{n \times n}$ and $F \geq 0$ (second term of the solution $x_{L}(t)$ ). Then $(F-V) \in M_{E}^{n \times n}$ and $(-V) \in M_{E}^{n \times n}$ and $F \geq 0$ are both sufficient conditions for $x_{L}(0) \succeq 0 \Longrightarrow x_{L}(t) \geq 0$; $\forall t \in \mathbf{R}_{0+}$. The necessity of $(F-V) \in M_{E}^{n \times n}$ follows by contradiction. If $(F-V) \notin M_{E}^{n \times n}$ then there exists at least one entry $i, j \in \bar{n}$ such that $\left(e^{(F-V) t}\right)_{i j}<0$ for some $t>0$. Then, it suffices to take $x_{L_{j}}(0)>0$ and $x_{L_{k}}(0)=0 ; \forall k(\neq$ $j) \in \bar{n}$ to conclude that $x_{L_{i}}(t)=-\left|\left(e^{(F-V) t}\right)_{i j}\right| x_{L_{j}}(0)<0$ so that the infective substate is not positive. Thus, $(F-V) \in$ $M_{E}^{n \times n}$ is a necessary and sufficient condition of nonnegativity of the solution of the infective linearized substate around the disease-free equilibrium point for all time and any nonnegative initial condition. Property (i) has been proved. Property (ii) is obvious from the fact that a necessary condition for nonnegativity of any solution under arbitrary nonnegative initial conditions is that the property holds for its linearized counterpart system. Property (iii) follows since, on one hand, the given conditions of Metzler matrices guarantee the nonnegativity of the linearized solution of the infective substates for any nonnegative initial conditions and all time. And, on the other hand, the convergence to the disease-free equilibrium of such a linearized subsystem follows from the stability of the Metzler matrices under the nonsingularity and positivity of their negative $M$-matrices counterparts according to Theorem A.1 [(i) to (iii)] and Theorem A.2 (ii) of Appendix A. Property (iv) is a dual version for instability of the sufficiency parts of Property (iii).

Note that if $(F-V) \in M_{E}^{n \times n}$ for any given $F \geq 0$ then $(-V) \in M_{E}^{n \times n}$ (otherwise, $(F-V) \notin M_{E}^{n \times n}$ if $F=0$ ). Therefore, $\left[(F-V) \in M_{E}^{n \times n} ; \forall F \geq 0\right] \Longleftrightarrow[(-V) \epsilon$ $\left.M_{E}^{n \times n} \wedge F \geq 0\right]$. This equivalence is reasonable, in practice, since the nonnegativity of the linearized trajectory solution around the disease-free equilibrium point of the infective compartment is also needed for zero reproduction numbers or zero coefficient transmission rates. The joint sufficiencytype condition of Theorem 1 (iii) is also necessary and this 
condition is equivalent to the other two Conditions (1)-(2). From Theorem 1 on the linearized infective substate and some further conditions on the nonlinear contribution of the dynamics in the model (1)-(3), we can obtain some further results for the nonnegativity of the solution and the stability of the whole nonlinear model as follows.

Theorem 2. The following properties hold.

(i) The state trajectory solution of (1)-(3) is nonnegative for all time, i.e., $z(t)=\left(x^{T}(t), y^{T}(t)\right)^{T} \geq 0 ; \forall t \in \mathbf{R}_{0+}$ for any given initial condition $z(0)=\left(x^{T}(0), y^{T}(0)\right)^{T} \geq 0$ if and only if the two subsequent constraints hold:

$$
\begin{aligned}
& \int_{0}^{t} f(x(\tau), y(\tau)) d \tau \preceq e^{(F-V) t} x(0) ; \quad \forall t \in \mathbf{R}_{0+} \\
& \int_{0}^{t} g(x(\tau), y(\tau)) d \tau \succeq-y(0) ; \quad \forall t \in \mathbf{R}_{0+} .
\end{aligned}
$$

Furthermore, one has that

$$
\begin{aligned}
& \limsup _{t \rightarrow \infty}\left(\int_{0}^{t} f(x(\tau), y(\tau)) d \tau-e^{(F-V) t} x(0)\right) \preceq 0 ; \\
& \liminf _{t \rightarrow \infty}\left(y(0)+\int_{0}^{t} g(x(\tau), y(\tau)) d \tau\right) \geq 0 .
\end{aligned}
$$

(ii) Assume, furthermore, that $(F-V) \in M_{E}^{n \times n}$ is a stability matrix or that any of the two constraints (1) or (2) of Theorem 1 (iii) holds. Then,

$$
\begin{aligned}
\int_{0}^{\infty} f(x(\tau), y(\tau)) d \tau & =0, \\
f(x(t), y(t)) & \longrightarrow 0 \text { as } t \longrightarrow \infty
\end{aligned}
$$

provided that $f:\left(\mathbf{R}_{0+}^{n} \times \mathbf{R}_{0+}\right) \times\left(\mathbf{R}_{0+}^{m} \times \mathbf{R}_{0+}\right) \longrightarrow \mathbf{R}_{0+}^{n}$ is everywhere uniformly continuous in its definition domain and $f(x(t), y(t)) \geq 0 ; \forall t \in \mathbf{R}_{0+} ;$ and $f(x(t), y(t)) \longrightarrow 0$ as $t \longrightarrow \infty$ (but nonnecessarily $\int_{0}^{\infty} f(x(\tau), y(\tau)) d \tau=0$ ) if $f:\left(\mathbf{R}_{0+}^{n} \times \mathbf{R}_{0+}\right) \times\left(\mathbf{R}_{0+}^{m} \times \mathbf{R}_{0+}\right) \longrightarrow \mathbf{R}_{0+}^{n}$ is everywhere uniformly continuous in its definition domain.

Proof. Property (i) follows directly from (1)-(3). On the other hand, if $(F-V) \in M_{E}^{n \times n}$, and $f(x(t), y(t)) \geq 0 ; \forall t \in$ $\mathbf{R}_{0+}$ and it is everywhere uniformly continuous for all time for its arguments in the first closed orthant of $\mathbf{R}^{n+m}$ for all time; then it follows that $\int_{0}^{\infty} f(x(\tau), y(\tau)) d \tau=0$, and $e^{(F-V) t} x(0) \longrightarrow 0$ and $f(x(t), y(t)) \longrightarrow 0$ as $t \longrightarrow \infty$. Also, if $f(x(t), y(t))$ is everywhere continuous for all time in $\mathbf{R}^{n+m}$ and $0=\lim _{t \longrightarrow \infty} e^{(F-V) t} \succeq \lim _{t \rightarrow \infty}\left|\int_{0}^{t} f(x(\tau), y(\tau)) d \tau\right|$ since $(F-V) \in M_{E}^{n \times n}$ and it is a stability matrix. Then, it follows from Barbalat's lemma $[18,24]$ that $f(x(t), y(t)) \rightarrow 0$ as $t \longrightarrow \infty$. Property (ii) has been proved.

Generally speaking, Theorem 1 (iv.(3)) is also guaranteed under sufficient conditions if the involved spectral radii [15, $25,26]$ are replaced by matrix norms which are their upperbounds. Also, note that Theorem 2 (ii) has the assumption that $(F-V) \in M_{E}^{n \times n}$ is stable which is needed to guarantee that no eigenvalue crosses the imaginary axis under perturbations of $F$ resulting in $(F-V)$ to lose the stability of $(F-V)$ when $F=0$, i.e., for $F-V=-V$. In the following discussions $(-V)$ is the transition matrix in-between state of the linearized subsystem of the infected variables around the disease-free equilibrium point while $F$ is the transmission matrix around such an equilibrium point.

On the other hand, it turns out that the nonnegativity condition $\int_{0}^{t} f(x(\tau), y(\tau)) d \tau \preceq e^{(F-V) t} x(0) ; \forall t \in \mathbf{R}_{0+}$ of Theorem 2 for the whole trajectory solution, subject to nonlinear dynamics, of the infective substate does not require that $(F-V) \in M_{E}^{n \times n}$ is a stability matrix or any conditions on the nonlinear term $f(x(t), y(t))$. Note that the condition holds directly if $-f(x(t), y(t)) \geq 0$ since then $0 \geq-e^{(F-V) t} x(0)$; $\forall x(0) \in \mathbf{R}_{0+}^{n}$ so that $-\int_{0}^{t} f(x(\tau), y(\tau)) d \tau \succeq 0 \geq-e^{(F-V) t} x(0)$. The nonnegativity of the trajectory solution can be also easily tested on the differential system (1)-(3) by ensuring that, for any zero value of a state component, its corresponding timederivative is nonnegative. So, we have the subsequent result which is alternative to the two first constraints of Theorem 2 (i).

\section{Theorem 3. The following properties hold.}

(i) The state trajectory solution of (1)-(3) is nonnegative for all time, i.e., $z(t)=\left(x^{T}(t), y^{T}(t)\right)^{T} \geq 0 ; \forall t \in \mathbf{R}_{0+}$ for any given initial condition $z(0)=\left(x^{T}(0), y^{T}(0)\right)^{T} \geq 0$ if and only if, for all $i \in \bar{n}, j \in \bar{m}$ and all $t \in \mathbf{R}_{0+}$, the following conditions hold:

$$
\begin{aligned}
& \left(x_{i}(t)=0\right) \Longrightarrow\left(f_{i}(x(t), y(t))\right. \\
& \left.\quad \leq \sum_{j(\neq i)=1}^{n}\left(F_{i j}-V_{i j}\right) x_{j}(t)=\sum_{j=1}^{n}\left(F_{i j}-V_{i j}\right) x_{j}(t)\right) \\
& \left(y_{j}(t)=0\right) \Longrightarrow\left(g_{j}(x(t), y(t)) \geq 0\right) .
\end{aligned}
$$

(ii) Property (i) also implies that $f_{i}\left(0, y_{d f}\right)=0 ; \forall i \in \bar{n}$, $g_{j}\left(0, y_{d f}\right)=0 ; \forall j \in \bar{m}$ if a disease-free equilibrium point $z_{d f}=\left(0^{T}, y_{d f}^{T}\right)^{T}$ exists.

Proof. It is obvious that, for any given $z(0) \geq 0$, if $z_{i}(t)=0$ for some $t \geq 0$, then $\dot{z}_{i}(t) \geq 0$ from the given conditions and it cannot give $\varepsilon \in \mathbf{R}_{+}$such that $z_{i}(t+\varepsilon)<0$ for some $i \in \bar{n}$. As a result, $z(t) \succeq 0 ; \forall t \in \mathbf{R}_{0+}$. The first part of the result has been proved. The second part is proved by contradiction. First note from the nonnegativity condition of Property (i) that $-f_{i}\left(0, y_{d f}\right) \geq-\left(\sum_{j=1}^{n}\left(F_{i j}-V_{i j}\right) .0\right)=0$. If $f_{i}\left(0, y_{d f}\right)=0$; $\forall i \in \bar{n}$ then Property (ii) has been proved for the infective substate. Now, assume that there exists some $i \in \bar{n}$ such that $f_{i}\left(0, y_{d f}\right)<0$. Since $\left.\vartheta(t)=\sum_{j=1}^{n}\left(F_{i j}-V_{i j}\right) x_{j}(t)\right]_{x(t)=0}=0$, it follows that $\left.\dot{x}_{i}\right]_{x_{i}=0}>0$ so that $z_{d f}=\left(0^{T}, y_{d f}^{T}\right)^{T}$ is not an equilibrium point; hence a contradiction follows. In the same way, if $g_{j}\left(0, y_{d f}\right)>0$ for some $j \in \bar{m}$ then $z_{d f}=$ $\left(0^{T}, y_{d f}^{T}\right)^{T}$ is not an equilibrium point; hence we have again a contradiction. Property (ii) has been proved.

Theorem 4. Let $g: \mathbf{R}^{n+m} \longrightarrow \mathbf{R}^{m}$ be everywhere continuously differentiable with a Jacobian matrix: 
$J_{g, z}\left(z_{0}\right)=\left[J_{g, x}\left(z_{0}\right) \mid J_{g, y}\left(z_{0}\right)\right]$ at any $z_{0}=\left(x_{0}^{T}, y_{0}^{T}\right)^{T} \epsilon$ $\mathbf{R}^{n+m}$ with $x_{0} \in \mathbf{R}^{n}$ and $y_{0} \in \mathbf{R}^{m}$, where $z=\left(x^{T}, y^{T}\right)^{T} \in \mathbf{R}^{n+m}$, $x \in \mathbf{R}^{n}, y \in \mathbf{R}^{m}$. Assume that the $m \times m$ matrix $J_{g, y}\left(z_{0}\right)$ is nonsingular at any equilibrium point $z_{0}$ of (1)-(3). Then, the following properties hold

(i) There exists an open set $U$ of $\mathbf{R}^{n}$ containing $x_{0}$ such that there exists a unique continuously differentiable function $h$ : $U \longrightarrow \mathbf{R}^{m}$ such that $y_{0}=h\left(x_{0}\right)$ and $f(x, h(x))=0 ; \forall x \in$ $U$. Furthermore, any equilibrium point $z_{0}=\left(x_{0}^{T}, y_{0}^{T}\right)^{T}$ can be expressed being dependent on the infective substate only, i.e., $z_{0}=\left(x_{0}^{T}, h^{T}\left(x_{0}\right)\right)^{T}$, while satisfying

$$
\begin{aligned}
& y_{0}=h\left(x_{0}\right) ; \\
& x_{0}=\left(F_{0}\left(h\left(x_{0}\right)\right)-V_{0}\left(x_{0}\right)\right)^{-1} f\left(x_{0}, h\left(x_{0}\right)\right)
\end{aligned}
$$

provided that $\left(F_{0}\left(h\left(x_{0}\right)\right)-V_{0}\left(x_{0}\right)\right)$ is nonsingular at any equilibrium point, where $F_{0}(h(0))=F$ and $V_{0}(0)=V$ if $z_{0}=z_{d f}=\left(0^{T}, h^{T}(0)\right)^{T}$ is the disease-free equilibrium point and $F_{0}\left(h\left(x_{\text {end }}\right)\right)=F_{e}$ and $V_{0}\left(x_{\text {end }}\right)=V_{e}$ if $z_{0}=z_{\text {end }}=$ $\left(x_{\text {end }}^{T}, h^{T}\left(x_{\text {end }}\right)\right)^{T}$ is the endemic equilibrium point.

(ii) Assume, furthermore, that the transition and transmission matrices of the disease-free equilibrium point satisfy that $(-V) \in M_{E}^{n \times n}$ is a stability matrix and $F \geq 0$. Then, (a) the disease-free equilibrium point exists and it is defined by $z_{d f}=\left(0^{T}, h^{T}(0)\right)^{T}$ and it is unique and locally asymptotically stable if $\rho\left(F V^{-1}\right)<1$. If $\rho\left(F V^{-1}\right)>1$ then it is unstable; and

(b) the endemic equilibrium point exists (while it is unique under the sufficiency-type conditions of Theorem B.2 of Appendix B) and it is defined by $z_{\text {end }}=\left(x_{\text {end }}^{T}, y_{\text {end }}^{T}\right)^{T}=$ $\left(x_{\text {end }}^{T}, h^{T}\left(x_{\text {end }}\right)\right)^{T}$ subject to

$$
\begin{aligned}
& y_{\text {end }}=h\left(x_{\text {end }}\right) \\
& x_{\text {end }}=\left(F_{e}-V_{e}\right)^{-1} f\left(x_{\text {end }}, h\left(x_{\text {end }}\right)\right)
\end{aligned}
$$

If it exists then it is reachable if $\rho\left(F V^{-1}\right) \geq 1$ and $f\left(x_{\text {end }}\right.$, $\left.h\left(x_{\text {end }}\right)\right) \preceq f\left(x_{d f}, h\left(x_{d f}\right)\right)$ while it is unreachable, in the sense that $z_{\text {end }} \notin \mathbf{R}_{0+}^{n+m}$, if $\rho\left(F V^{-1}\right)<1$ supposing that $f\left(x_{\text {end }}\right.$, $\left.h\left(x_{\text {end }}\right)\right)>f\left(x_{d f}, h\left(x_{d f}\right)\right)$ if $z_{\text {end }} \neq z_{d f}$.

Proof. Assume that $J_{g, y}\left(x_{0}, y_{0}\right) \in \mathbf{R}^{m} \times \mathbf{R}^{m}$ is nonsingular. Then, there exits an open set $U$ of $\mathbf{R}^{n}$ containing $x_{0}$ such that there exists a unique continuously differentiable function $h$ : $U \longrightarrow \mathbf{R}^{m}$ such that $y_{0}=h\left(x_{0}\right)$ and $f(x, h(x))=0 ; \forall x \in U$. Let $z_{0}$ be either the disease-free equilibrium point $z_{0}=z_{d f}=$ $\left(0^{T}, y_{0}^{T}\right)^{T}=\left(0^{T}, h^{T}(0)\right)^{T}$ or the endemic one $z_{0}=z_{\text {end }}=$ $\left(x_{\text {end }}^{T}, y_{\text {end }}^{T}\right)^{T}=\left(x_{\text {end }}^{T}, h^{T}\left(x_{\text {end }}\right)\right)$. Both of them are unique in a neighbourhood centred at the respective equilibrium point, since $h(\cdot)$ is unique, while they satisfy (10) and, respectively, (11) if $J_{g, z}\left(z_{d f}\right)$ and, respectively, $J_{g, z}\left(z_{\text {end }}\right)$ are nonsingular since $\left(F_{0}\left(x_{0}\right)-V_{0}\left(x_{0}\right)\right)$ is a nonsingular matrix. Property (i) has been proved. In order to prove Property (ii), first note that $(-V) \in M_{E}^{n \times n}$ is nonsingular with $V^{-1}>0$ (since $(-V)$ is stable) with $F \geq 0$. Then, the disease-free equilibrium point exists being defined by $z_{d f}=\left(0^{T}, h^{T}(0)\right)^{T}$; it is unique since $h(\cdot)$ is unique and given by $x_{d f}=(F-V)^{-1} f(0, h(0))=0$.
Furthermore, it is locally asymptotically stable if $\rho\left(F V^{-1}\right)<1$ since then $(F-V) \in M_{E}^{n \times n}$ is a stability matrix, equivalently $(V-F)^{-1}>0$ so that the linearized system around the disease-free equilibrium point is locally exponentially (then asymptotically) stable. On the other hand, it is unstable if $\rho\left(F V^{-1}\right)>1$ [Theorem 1 (iii)-(iv)]. The part (a) of Property (ii) has been proved. Now let $z_{0}=z_{\text {end }}=\left(x_{\text {end }}^{T}, y_{\text {end }}^{T}\right)^{T}$ be the endemic equilibrium point. The following cases can occur

(1) If $\rho\left(F V^{-1}\right) \geq 1$, then note that

$$
\begin{aligned}
x_{\text {end }} & =\left(V_{e}-F_{e}\right)^{-1}\left(-f\left(x_{\text {end }}, h\left(x_{\text {end }}\right)\right)\right) \\
& \geq(V-F)^{-1}\left(-f\left(x_{d f}, h\left(x_{d f}\right)\right)\right) \geq 0
\end{aligned}
$$

since $f\left(x_{\text {end }}, h\left(x_{\text {end }}\right)\right) \preceq 0$ (being a strict inequality if and only if $\left.x_{\text {end }} \neq x_{d f}=0\right)$ and $(F-V) \in M_{E}^{n \times n}$ is stable and then nonsingular

(2) If $R_{0}=\rho\left(F V^{-1}\right)<1$ then $(V-F)^{-1}>0$, since $(F-V) \epsilon$ $M_{E}^{n \times n}$, and $F_{e}>F$. Therefore, $\left(I_{n}+(V-F)^{-1}\left(F_{e}-F\right)\right)>0$ and $\rho\left[(V-F)^{-1}\left(F_{e}-F\right)\right]>0$ then $\left(I_{n}+\left(I_{n}-V^{-1} F\right)^{-1} V^{-1}\left(F_{e}-F\right)\right)^{-1}$ exists, and

$$
\begin{aligned}
\left(V_{e}\right. & \left.-F_{e}\right)^{-1}=\left(I_{n}+(V-F)^{-1}\left(F_{e}-F\right)\right)^{-1}(V-F)^{-1} \\
& =\left(I_{n}+\left(I_{n}-V^{-1} F\right)^{-1} V^{-1}\left(F_{e}-F\right)\right)^{-1}(V-F)^{-1} \\
& \prec(V-F)^{-1}
\end{aligned}
$$

Note that $x_{\text {end }}$ is unreachable if $x_{\text {end }} \neq x_{d f}=0$ since $-f\left(x_{\text {end }}, h\left(x_{\text {end }}\right)\right) \prec-f\left(x_{d f}, h\left(x_{d f}\right)\right)=0$, and

$$
\begin{aligned}
x_{\text {end }}= & \left(V_{e}-F_{e}\right)^{-1}\left(-f\left(x_{\text {end }}, h\left(x_{\text {end }}\right)\right)\right) \\
\prec & \left(I_{n}+\left(I_{n}-V^{-1} F\right)^{-1} V^{-1}\left(F_{e}-F\right)\right)^{-1} \\
& \times(V-F)^{-1}\left(-f\left(x_{d f}, h\left(x_{d f}\right)\right)\right) \\
= & \left(I_{n}+\left(I_{n}-V^{-1} F\right)^{-1} V^{-1}\left(F_{e}-F\right)\right)^{-1} x_{d f}=0 .
\end{aligned}
$$

Then, $x_{\text {end }} \preceq 0$. But, for the case, $x_{\text {end }}=0$, the endemic equilibrium point is coincident with the disease-free one, that is, $x_{\text {end }}=x_{d f}=0$ and $\rho\left(F V^{-1}\right)=1$, while, for $\rho\left(F V^{-1}\right)<1$, $x_{\text {end }} \prec 0$,; hence a contradiction to its reachability follows since the equilibrium point is never allocated in the open first orthant of $\mathbf{R}^{n}$. Property (ii) has been proved.

Remark 5. It has not been proved that the endemic equilibrium point is necessarily unique, independently of the concrete epidemic model, in the whole state space. A detailed proof of the global uniqueness of the disease-free equilibrium point is given in Theorem B.1 (i) of Appendix B under the same conditions as those given in Theorem 4 (ii) since the infective equilibrium substate is unique and identically zero what guarantees also the uniqueness of the nondisease compartment via the uniqueness of the function $h(\cdot)$. On the other hand, the endemic equilibrium point $z_{\text {end }}$ is unique in the whole state space if $x_{\text {end }}$, i.e., its infective substate, 
is unique since from the uniqueness of $h(\cdot)$, the uniqueness of the nondisease compartment and then that of the whole endemic equilibrium point are also guaranteed under some algebraic conditions given in Theorem B.2 in Appendix B.

It is well-known from the background literature on epidemic models, subject to a unique disease-free equilibrium point and a unique endemic equilibrium one, that, typically, if the reproduction number $R_{0}<1$ then the disease-free equilibrium point is asymptotically stable while the endemic one is not reachable since it has negative infective values what is incompatible with the positivity of the state trajectory solution. If $R_{0}>1$ then the endemic equilibrium point is stable and the disease-free one is unstable and, if $R_{0}=1$, then the infective variables of both equilibrium points are coincident. Theorem 4 describes formally via algebraic tools the reachability/unreachability of the endemic equilibrium point depending on the value of the reproduction number. This fact is illustrated by the subsequent example.

Example 6. A particular case of the SEIADR (susceptibleexposed-symptomatic infectious-asymptomatic infectiousinfectious corpses-recovered) proposed in $[6,7]$ follows below for the case when the vaccination and treatment control parameters $V_{0}, K_{V}$, and $K_{\xi}$ are constant and there is no impulsive control for the infectious corpses removal control action:

$$
\begin{aligned}
\dot{S}(t)= & b_{1}-\left(b_{2}+\beta I(t)+\beta_{A} A(t)+\beta_{D} D(t)\right) S(t) \\
& +\eta R(t)-V(t) \\
\dot{E}(t)= & -\left(b_{2}+\gamma\right) E(t) \\
& +\left(\beta I(t)+\beta_{A} A(t)+\beta_{D} D(t)\right) S(t) \\
\dot{I}(t)= & -\left(b_{2}+\alpha+\tau_{0}+K_{\xi}\right) I(t)+\gamma p E(t) \\
\dot{A}(t)=- & \left(b_{2}+\tau_{0}\right) A(t)+\gamma(1-p) E(t) \\
\dot{D}(t)= & -\mu D(t)+b_{2}(I(t)+A(t))+\alpha I(t) \\
\dot{R}(t)=- & \left(b_{2}+\eta\right) R(t)+\tau_{0}(I(t)+A(t))+K_{\xi} I(t) \\
& +V(t) \\
V(t)= & V_{0}+K_{V} S(t)
\end{aligned}
$$

where $V(t)$ is a vaccination control of constant gain $V_{0}$ plus a linear feedback term with gain $K_{V}$ with information of the susceptible subpopulation. There is also an eventual treatment control on the infectious subpopulation of gain $K_{\xi}$. The disease-free and endemic Jacobian matrices for the joint noninfective infective dynamically coupled compartments are, respectively,

$$
\mathbf{A}_{d f}=\left[\begin{array}{cccccc}
-\left(b_{2}+K_{V}\right) & 0 & -\beta S_{d f} & -\beta_{A} S_{d f} & -\beta_{D} S_{d f} & \eta \\
0 & -\left(b_{2}+\gamma\right) & \beta S_{d f} & \beta_{A} S_{d f} & \beta_{D} S_{d f} & 0 \\
0 & \gamma p & -\left(b_{2}+\alpha+\tau_{0}+K_{\xi}\right) & 0 & 0 & 0 \\
0 & \gamma(1-p) & 0 & -\left(b_{2}+\tau_{0}\right) & 0 & 0 \\
0 & 0 & b_{2}+\alpha & b_{2} & -\mu & 0 \\
K_{V} & 0 & \tau_{0}+K_{\xi} & \tau_{0} & 0 & -\left(b_{2}+\eta\right)
\end{array}\right]
$$

where the infective compartment has a Jacobian matrix $F-V$ uncoupled to the noninfective one with

$$
V=\left[\begin{array}{cccc}
b_{2}+\gamma & 0 & 0 & 0 \\
-\gamma p & b_{2}+\alpha+\tau_{0}+K_{\xi} & 0 & 0 \\
-\gamma(1-p) & 0 & b_{2}+\tau_{0} & 0 \\
0 & -\left(b_{2}+\alpha\right) & -b_{2} & \mu
\end{array}\right]
$$

$$
F=\beta\left[\begin{array}{cccc}
0 & S_{d f} & \beta_{A r} S_{d f} & \beta_{D r} S_{d f} \\
0 & 0 & 0 & 0 \\
0 & 0 & 0 & 0 \\
0 & 0 & 0 & 0
\end{array}\right]
$$

and

$$
A_{\text {end }}=\left[\begin{array}{cccccc}
-\left(b_{2}+\beta I_{\text {end }}+\beta_{A} A_{\text {end }}+\beta_{D} D_{\text {end }}+K_{V}\right) & 0 & -\beta S_{\text {end }} & -\beta_{A} S_{\text {end }} & -\beta_{D} S_{\text {end }} & \eta \\
\beta I_{\text {end }}+\beta_{A} A_{\text {end }}+\beta_{D} D_{\text {end }} & -\left(b_{2}+\gamma\right) & \beta S_{\text {end }} & \beta_{A} S_{\text {end }} & \beta_{D} S_{\text {end }} & 0 \\
0 & \gamma p & -\left(b_{2}+\alpha+\tau_{0}+K_{\xi}\right) & 0 & 0 & 0 \\
0 & \gamma(1-p) & 0 & -\left(b_{2}+\tau_{0}\right) & 0 & 0 \\
0 & 0 & b_{2}+\alpha & b_{2} & -\mu & 0 \\
K_{V} & 0 & \tau_{0}+K_{\xi} & \tau_{0} & 0 & -\left(b_{2}+\eta\right)
\end{array}\right]
$$


where the infective compartment Jacobian matrix is $F_{e}-V_{e}$ with

$$
\begin{aligned}
& V_{e}=V=\left[\begin{array}{cccc}
b_{2}+\gamma & 0 & 0 & 0 \\
-\gamma p & b_{2}+\alpha+\tau_{0}+K_{\xi} & 0 & 0 \\
-\gamma(1-p) & 0 & b_{2}+\tau_{0} & 0 \\
0 & -\left(b_{2}+\alpha\right) & -b_{2} & \mu
\end{array}\right] \\
& F_{e}=\beta\left[\begin{array}{cccc}
0 & S_{\text {end }} & \beta_{A r} S_{\text {end }} & \beta_{D r} S_{\text {end }} \\
0 & 0 & 0 & 0 \\
0 & 0 & 0 & 0 \\
0 & 0 & 0 & 0
\end{array}\right]=F \\
& -\beta\left[\begin{array}{cccc}
0 & S_{d f}-S_{\text {end }} & \beta_{A r}\left(S_{d f}-S_{\text {end }}\right) & \beta_{D r}\left(S_{d f}-S_{\text {end }}\right) \\
0 & 0 & 0 & 0 \\
0 & 0 & 0 & 0 \\
0 & 0 & 0 & 0
\end{array}\right] \\
& =F-\beta\left(R_{0}-1\right) S_{\text {end }}\left[\begin{array}{cccc}
0 & 1 & \beta_{A r} & \beta_{D r} \\
0 & 0 & 0 & 0 \\
0 & 0 & 0 & 0 \\
0 & 0 & 0 & 0
\end{array}\right]
\end{aligned}
$$

with $R_{0}=\rho\left(F V^{-1}\right)=S_{d f} / S_{\text {end }}$. Note that $F_{e} \geq F$ if $R_{0} \leq 1$; $F \geq F_{e}$ if $R_{0} \geq 1$; and $F=F_{e}$ if $R_{0}=1$ (what implies $x_{\text {end }}=$ $\left.x_{d f}\right)$, and

$$
\begin{aligned}
& f\left(0, y_{d f}\right)=0 ; \\
& -f\left(x_{\text {end }}, y_{\text {end }}\right) \\
& \quad=\left[\begin{array}{c}
\left(\beta I_{\text {end }}+\beta_{A} A_{\text {end }}+\beta_{D} D_{\text {end }}\right) S_{\text {end }} \\
0 \\
0 \\
0
\end{array}\right] \geq 0
\end{aligned}
$$

irrespective that the infective variables be positive or negative since $I_{\text {end }}, A_{\text {end }}$, and $D_{\text {end }}$ have the same sign as $E_{\text {end }}$, if $E_{\text {end }} \neq$ 0 , that is, if the endemic equilibrium point has not coincident components with the disease-free one, that is, if $R_{0} \neq 1$, as it is deduced from the epidemic model. Note from the matrix $\mathbf{A}_{d f}$ that if the linearized infective subsystem around the diseasefree equilibrium is identically zero, then the characteristic equation of the linearized noninfective subsystem is given by

$$
p_{n i}(s)=s^{2}+\left(2 b_{2}+K_{V}+\eta\right) s+b_{2}\left(b_{2}+\eta+K_{V}\right)=0
$$

whose roots are $s_{1}=-b_{2}<0$ and $s_{2}=-\left(b_{2}+K_{V}+\eta\right)<$ 0 . So, such a subsystem is asymptotically stable satisfying Assumption 2(a). As a result, the whole linearized subsystem is asymptotically stable if $R_{0} \leq 1$.

It has been proved in Theorem 4 that the disease-free equilibrium point is stable if $\rho\left(F V^{-1}\right) \leq 1$, the endemic one is unattainable if $\rho\left(F V^{-1}\right)<1$, and the disease-free equilibrium point is unstable if $\rho\left(F V^{-1}\right)>1$ while the endemic one is reachable if $\rho\left(F V^{-1}\right) \geq 1$. The next result establishes that the endemic equilibrium point is unstable when unattainable and asymptotically stable when attainable.

Proposition 7. Assume the following.

(1) The endemic and the disease-free transition matrices $\left(-V_{e}\right)$ and $(-V)$ are identical and independent of the reproduction number, and $(-V) \in M_{E}^{n \times n}$ is a stability matrix.

(2) The disease-free and endemic equilibrium points exist and they are unique for any $R_{0} \in[0,+\infty]$.

(3) The disease-free and the endemic transmission matrices are nonnegative, i.e., $F=F\left(R_{0}\right) \geq 0$, and $F_{e}=F_{e}\left(R_{0}\right)=$ $\varsigma\left(R_{0}\right) F \geq 0$, where $R_{0}=R_{0}(F)=\rho\left(F V^{-1}\right)$ is the reproduction number, and $\varsigma\left(R_{0}\right) \geq 0$.

(4) $F-F_{e}=(\lambda-1) F \geq 0$ for some $\lambda \in \mathbf{R}$.

(5) $F>F_{e}$ if $R_{0}>1, F \prec F_{e}$ if $R_{0}<1$, and $F=F_{e}$ if $R_{0}=1$.

Then, the endemic equilibrium point is locally asymptotically stable if $R_{0} \geq 1$ and unstable if $R_{0}<1$.

Proof. Note that

$$
F-V=\left(I_{n}-F V^{-1}\right)(-V)
$$

Since $(-V)$ is a stability matrix, the disease-free equilibrium point is locally asymptotically stable if $R_{0}=\rho\left(F V^{-1}\right)<1$ and unstable if $R_{0}=\rho\left(F V^{-1}\right)>1$, the critical stability case being $R_{0}=\rho\left(F V^{-1}\right)=1$. Note also that

$$
\begin{aligned}
F_{e}-V & =\left(I_{n}-F_{e} V^{-1}\right)(-V) \\
& =\left(I_{n}-F V^{-1}+\left(F-F_{e}\right) V^{-1}\right)(-V) .
\end{aligned}
$$

Since $F-F_{e}=(\lambda-1) F \geq 0$ with $\lambda=\lambda\left(R_{0}\right) \geq 1$ for $R_{0} \leq 1$ since $F \geq F_{e}$, then $\rho\left(F-F_{e}\right)=(\lambda-1) \rho(F) \geq 0$ and both of them are proportional matrices because of their structure, it follows that the endemic equilibrium point is unstable for $R_{0}<1$ (when the disease-free is locally stable ) and locally stable for $R_{0}>1$ (when the disease-free one is unstable).

Note that for Example 6, $\lambda\left(R_{0}\right)=2-1 / R_{0}$ in the proof of Proposition 7. Note that assumption 4 of Proposition 7 is very reasonable if $F$ and $F_{e}$ are related by a scalar factor which is the usual case in epidemic models. The subsequent examples show that the endemic equilibrium is unstable in the unattainable region.

Example 8. Consider the SEIADR epidemic model of Example 6 with the following parameterization:

$$
\begin{aligned}
& b_{1}=b_{2}=1 / 25550 \text { days }^{-1} ; 1 / \tau_{0}=15.8 \text { days } ; \eta=2 \mu= \\
& 0.1 \text { days }^{-1} ; \\
& p=0.15 ; 1 / \gamma=15.8 \text { days } ; 1 / \alpha=13.3 \text { days } ; \\
& V_{0}=b_{1} ; K_{V}=20 b_{1} ; K_{\xi}=\alpha ; \\
& \beta=0.11 \text { days }^{-1} ; \beta_{A}=0.05 \text { days }^{-1} ; \beta_{D}=0.15 \text { days }^{-1} .
\end{aligned}
$$

Figure 1 shows the maximum of the real parts of the eigenvalues of the Jacobian around the disease-free and endemic equilibrium points versus the reproduction number. In the proposed example, they coincide with the maximum eigenvalue since it is real. The reproduction number is displayed 


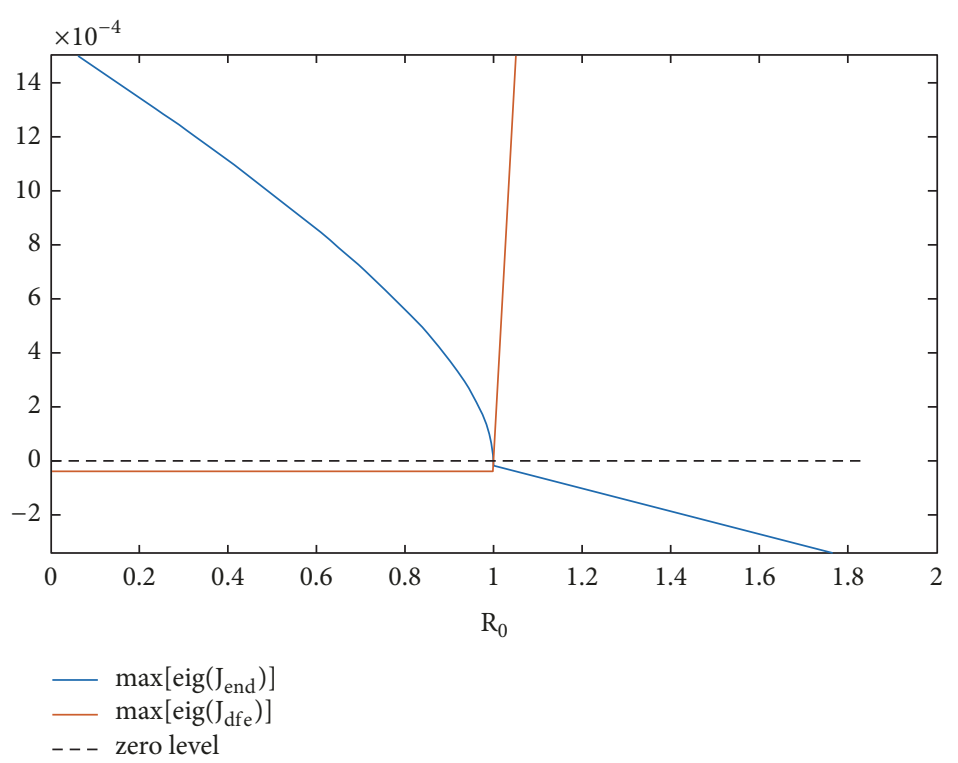

Figure 1: Maximum Eigenvalues of the Jacobians of the model of Example 6.

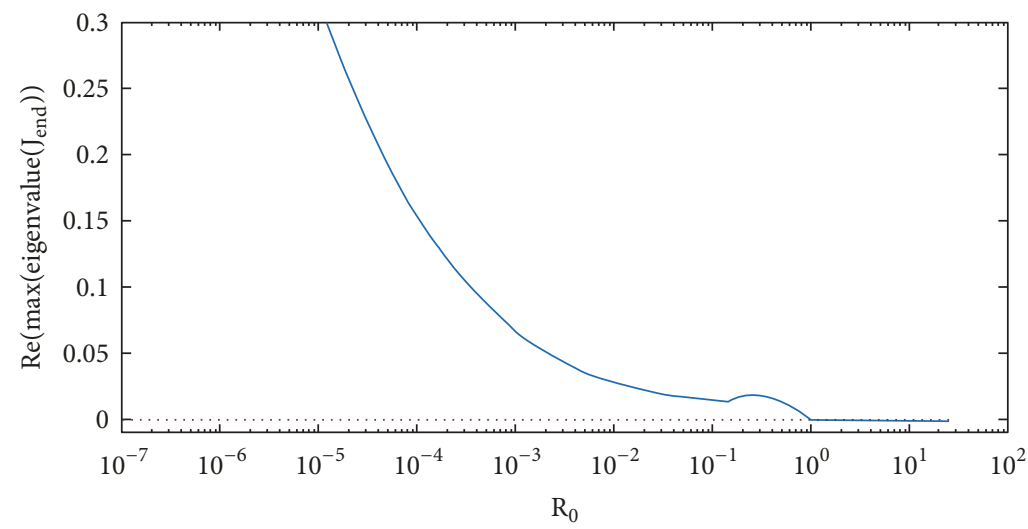

FIgURE 2: Maximum real part of the eigenvalues versus the reproduction number.

in the real axis by keeping constant all the disease and control model parameters except the coefficient transmission rates which are modified from their given basic values proportionally via the same common direct proportionality constant. Note that the reproduction number is proportional to the disease coefficient rate if all the remaining parameters are kept constant. It is seen in Figure 1 that the value $R_{0}=1$ is the critical reproduction number giving the frontier between the stability (instability) of the disease-free equilibrium point together with the unattainability and instability (attainability and stability) of the endemic one.

Example 9. Consider a true mass action (i.e., the nonlinear infective terms of susceptible-infected products are normalized with the total population) variant of the SEIADR epidemic model of Examples 6 and 8 with the parameterization of Example 8. Figure 3 shows a zoom of Figure 2 centred in the stability boundary examined on the maximum eigenvalue evolution of the Jacobian of the endemic equilibrium point. It is seen that the endemic equilibrium is unstable in its unattainable region under nonnegativity conditions of the solution while the disease-free one is the unique asymptotically stable attractor if the reproduction number is less than one. However, if such a reproduction number exceeds unity, then the disease-free equilibrium point is unstable while the endemic one is reachable and asymptotically stable.

A global stability theorem now follows by combining the preceding results and the analysis of Poincaré indices and the alternate stability characteristics of limit cycles surrounding singular points in any hyperplanes of the state space. It is proved, in particular, that the local asymptotic stability of the disease-free equilibrium point for a reproduction number less than one (implying also the unattainability of the endemic equilibrium point) leads to the global asymptotic stability of the whole state towards the disease-free equilibrium point. Note that Proposition 7 concludes that only one of the equilibrium points is locally stable for each given value of $R_{0}$. In particular the disease-free one is locally asymptotically stable if $R_{0} \leq 1$ and the endemic one is locally asymptotically 


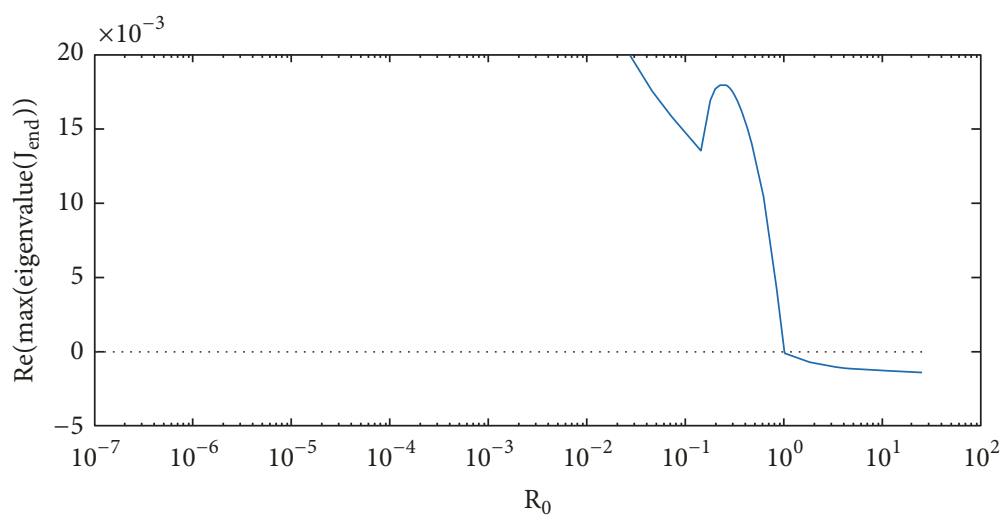

FIGURE 3: Zoom of the maximum real part of the eigenvalues versus the reproduction number close to the stability region boundary.

stable if $R_{0} \geq 1$. Note also that Proposition 7 agrees with the conclusions of Examples 6-9.

Theorem 10. Under Assumptions 1-2, assume also that $(-V) \in$ $M_{E}^{n \times n}, V^{-1}>0, F \geq 0$, and $\rho\left(F V^{-1}\right)<1$. Moreover, assume that (1) $J_{g, z}\left(\begin{array}{ll}0 & y_{d f}^{T}\end{array}\right)$ is nonsingular,

(2) $g_{i}(z(t)) \geq 0$ if $y_{i}(t)=0$ for any $i \in \bar{m}$ and $t \in \mathbf{R}_{0+}$,

(3) $f_{i}(z(t)) \leq e_{i}^{T}(F-V) x(t)$ if $x_{i}(t)=0$ for any $i \in \bar{n}$ and $t \in \mathbf{R}_{0+}$ (it suffices that $f_{i}(z(t)) \leq 0$ if $x_{i}(t)=0$ for any $i \in \bar{n}$ ).

(i) Then, the total population, i.e., the sum of all the subpopulations, is uniformly bounded for all time. Furthermore, any trajectory solution is nonnegative and uniformly bounded for all time for any given finite initial condition $x(0) \geq 0$ and $y(0) \geq 0$ while it is globally convergent at an exponential rate to the disease-free equilibrium point which is the unique reachable equilibrium point which is a globally asymptotically stable attractor.

(ii) Assume that for $R_{0}>1$ the total population, i.e., the sum of all the subpopulations, is uniformly bounded for all time. Assume also that $f(x(t), y(t))$ and $g(x(t), y(t))$ are uniformly continuous in the first closed orthant of $\mathbf{R}^{n+m}$. Then, all the trajectory solutions are bounded for all time while they converge asymptotically to the endemic equilibrium point at an exponential rate which is also the unique asymptotically stable attractor.

Proof. The nonnegativity of the solutions and the local asymptotic stability of the disease-free equilibrium point, which is unique, follow directly from Theorem 1 (iii) and Theorem 4 (ii. (a)), since from Theorem 4 (ii. (b)), the endemic equilibrium is not reachable if $\rho\left(F V^{-1}\right)<1$. Since the unique disease-free equilibrium point is locally asymptotically stable, its Poincaré index [17] is +1 in any plane of the phase space containing the evolution of any two components of the state trajectory solution. It is wellknown from the existence theorem of oscillations that any such a solution, if it exists, should be bounded. This is a consequence of the fact that the total population is bounded for all time and that all the subpopulations are nonnegative for all time what leads to the conclusion that any of them is uniformly bounded for all time for any finite initial conditions in the first closed orthant of $\mathbf{R}^{n+m}$. Also, any such an oscillation, if it exists, should surround the equilibrium point, since the net global Poincaré index of all the reachable equilibrium points is still +1 , while it should be unstable and then asymptotically vanishing to the locally asymptotically stable disease-free equilibrium point, since such a unique equilibrium point within the region defined by such a curve, is locally asymptotically stable. As a result, the disease-free equilibrium point is globally asymptotically stable for any initial condition in the first closed orthant of $\mathbf{R}^{n+m}$. Property (i) has been proved. Now, assume that the total population is bounded for $R_{0}>1$ so that the disease-free equilibrium point is unstable and the endemic one is attainable. Two cases can occur.

Case $a$. The endemic equilibrium point is globally asymptotically stable. Property (ii) follows directly.

Case $b$. The endemic equilibrium point is not globally asymptotically stable. Since the total population is bounded by hypothesis and all the subpopulations are nonnegative for all time, all the subpopulations are bounded for all time and a bounded limit cycle, if it exists, should surround the endemic point to attract any trajectories in the first orthant of the state space. This consideration follows by examining the admissible Poincaré-type combinations of allowed stable/instable combinations of configurations of singular points and limit cycles. Rewrite compactly (1) to (3) in the form $\dot{z}(t)=\widehat{f}(x(t), y(t))$, where $\widehat{f}(x(t), y(t))=\left[\begin{array}{cc}F-V & 0 \\ 0 & 0\end{array}\right]\left[\begin{array}{l}x(t) \\ y(t)\end{array}\right]+$ $\left[\begin{array}{c}-f(x(t), y(t)) \\ g(x(t), y(t))\end{array}\right]$. Assume that $\int_{0}^{\infty} \widehat{f}(x(\tau), y(\tau)) d \tau= \pm \infty$. Then, $z(t) \longrightarrow \pm \infty$ as $t \longrightarrow \infty$ which is not compatible with the nonnegativity of the solution trajectories within the first orthant and their boundenness. Therefore, $-\infty<$ $\int_{0}^{\infty} \widehat{f}(x(\tau), y(\tau)) d \tau<+\infty$. Since $\widehat{f}(x(\cdot) \cdot y(\cdot))$ is uniformly continuous, it follows from Barbalat's lemma, [24], that $\dot{z}(t) \longrightarrow 0$ as $t \longrightarrow \infty$ so that $z(t)$ cannot have a limit oscillation in any of its components. So, a stable limit cycle cannot surround the endemic equilibrium point if unstable or critically stable. Therefore, the endemic equilibrium point is globally asymptotically stable so that $z(t) \longrightarrow z_{\text {end }}$ as $t \longrightarrow$ $\infty$. Property (ii) has been proved. 
A very important remark is that the mathematical hypothesis of boundedness of the total population for the cases of reproduction numbers exceeding unity of Theorem 10 (ii) is not strong, then fully feasibly, in practice. This consideration is based on the proved boundedness of the total population in the case that the reproduction number is less than unity implying the global asymptotic stability. In fact, it can be conjectured from simple inspection that, for any given initial conditions in the first orthant and any given time instant, the total population cannot exceed its value for the disease-free case at any time because thereof the disease mortality rates. Even if there is no infection mortality both values would be coincident.

\section{Models with Multiple Incommensurate Point Time-Delays}

A compartmental disease model of dimension $(m+n)$ with $r$ constant, in general incommensurate, internal point delays $h_{i}(i \in \bar{r})$ satisfying $0=h_{0} \leq h_{1} \leq h_{2} \leq \cdots \leq h_{r}=h \leq \bar{h} \leq$ $\infty$, what is assumed in the sequel, can be generalized from (1)-(2) as follows:

$$
\begin{aligned}
& \dot{x}(t)=\sum_{i=0}^{r}\left(F_{i}\left(x\left(t-h_{i}\right), y\left(t-h_{i}\right)\right)\right. \\
& \left.\quad-V_{i}\left(x\left(t-h_{i}\right), y\left(t-h_{i}\right)\right)\right)=\sum_{i=0}^{r}\left(F_{i}-V_{i}\right) x(t \\
& \left.\quad-h_{i}\right)-f\left(x(t), y(t), x\left(t-h_{1}\right), y\left(t-h_{1}\right), \ldots, x(t\right. \\
& \left.\left.\quad-h_{r}\right), y\left(t-h_{r}\right)\right) \\
& \dot{y}(t) \equiv g(\widehat{x}(t), \hat{y}(t))=g\left(x(t), y(t), x\left(t-h_{1}\right), y(t\right. \\
& \left.\left.\quad-h_{1}\right), \ldots, x\left(t-h_{r}\right), y\left(t-h_{r}\right)\right)
\end{aligned}
$$

subject to any absolutely continuous function of initial conditions $\varphi:[-h, 0] \longrightarrow \mathbf{R}^{n+m}$ with eventual finite jumps on a subset of $[-h, 0]$ of zero measure with $\varphi(0)=\left(x^{T}(0), y^{T}(0)\right)^{T}$. Any member of such a class of functions is referred to in the sequel as an admissible function of initial conditions, where $n$ and $m$ are the respective dimensions of the disease and nondisease compartments of respective state vectors $x(t)$ and $y(t)$, and the nonlinear effects of (3) are replaced by the subsequent delayed counterpart dynamics:

$$
\begin{aligned}
& f(\widehat{x}(t), \widehat{y}(t)) \equiv f\left(x(t), y(t), x\left(t-h_{1}\right), y\left(t-h_{1}\right),\right. \\
& \left.\quad \ldots, x\left(t-h_{r}\right), y\left(t-h_{r}\right)\right)=\sum_{i=0}^{r}\left(F_{i}-V_{i}\right) x\left(t-h_{i}\right) \\
& \quad-\sum_{i=0}^{r}\left(F_{i}\left(x\left(t-h_{i}\right), y\left(t-h_{i}\right)\right)\right. \\
& \left.-V_{i}\left(x\left(t-h_{i}\right), y\left(t-h_{i}\right)\right)\right)=\theta(\widehat{z}(t)) G(\widehat{z}(t))
\end{aligned}
$$

where $f(\widehat{x}(t), \widehat{y}(t))$ describes the dynamics of higher-order contributions to the dynamics, $\widehat{z}(t)=\left(\widehat{x}^{T}(t), \widehat{y}^{T}(t)\right)=$ $\left(z^{T}(t), z^{T}\left(t-h_{1}\right), \ldots, z^{T}\left(t-h_{r}\right)\right)$, where $z(t)=\left(y^{T}(t), x^{T}(t)\right)^{T}$ and $\theta(\widehat{z}(t))$ is the scalar positive bounded incidence rate, and $G(\widehat{z}(t))=\sum_{i=0}^{r} F_{i}\left(z\left(t-h_{i}\right)\right) z\left(t-h_{i}\right)$ where $G(\widehat{z}(t))$ is a partitioned block matrix function of the form $G(\widehat{z}(t))=$ $\sum_{i=0}^{r}\left[G_{x x}\left(x\left(t-h_{i}\right)\right) G_{x y}\left(y\left(t-h_{i}\right)\right)\right]$. For commensurate point delays, (24)-(26) remain valid under the additional constraint $h_{i}=i h_{1}$ for $i \in \bar{r}$. It turns out that the solution is unique on $[-h, \infty)$ for any such initial conditions from the CauchyPeano theorem. Note that, (24)- (26) can be rewritten as follows:

$$
\begin{aligned}
\dot{x}(t)= & \sum_{i=0}^{r}\left(F_{i}-V_{i}\right) x\left(t-h_{i}\right)-f(\widehat{x}(t), \widehat{y}(t)) \\
= & \left(F_{0}-V_{0}\right) x(t)+\sum_{i=1}^{r}\left(F_{i}-V_{i}\right) x\left(t-h_{i}\right) \\
& -f(\widehat{x}(t), \widehat{y}(t)) \\
= & (F-V) x(t)+\sum_{i=1}^{r}\left(F_{i}-V_{i}\right)\left(x\left(t-h_{i}\right)-x(t)\right) \\
& -f(\widehat{x}(t), \widehat{y}(t)) \\
\dot{y}(t) \equiv & g(\widehat{x}(t), \widehat{y}(t))
\end{aligned}
$$

where

$$
\begin{aligned}
f( & \widehat{x}(t), \widehat{y}(t))=\sum_{i=0}^{r}\left(F_{i}-V_{i}\right) x\left(t-h_{i}\right) \\
& -\sum_{i=0}^{r}\left(F_{i}\left(x\left(t-h_{i}\right), y\left(t-h_{i}\right)\right)\right. \\
& \left.-V_{i}\left(x\left(t-h_{i}\right), y\left(t-h_{i}\right)\right)\right)=\left(F_{0}-V_{0}\right) x(t) \\
& +\sum_{i=1}^{r}\left(F_{i}-V_{i}\right) x\left(t-h_{i}\right) \\
& -\sum_{i=0}^{r}\left(F_{i}\left(x\left(t-h_{i}\right), y\left(t-h_{i}\right)\right)\right. \\
& \left.-V_{i}\left(x\left(t-h_{i}\right), y\left(t-h_{i}\right)\right)\right)=\left(F_{0}-V_{0}\right) x(t) \\
& +\sum_{i=1}^{r}\left(F_{i}-V_{i}\right)\left(x\left(t-h_{i}\right)-x(t)\right) \\
& -\sum_{i=0}^{r}\left(F_{i}\left(x\left(t-h_{i}\right), y\left(t-h_{i}\right)\right)\right. \\
& \left.-V_{i}\left(x\left(t-h_{i}\right), y\left(t-h_{i}\right)\right)\right)
\end{aligned}
$$

with

$$
\begin{aligned}
& F_{j}=\left[\frac{\partial F_{j i}\left(0, y_{d f}\right)}{\partial x_{j}\left(t-h_{j}\right)}\right] ; \\
& V_{j}=\left[\frac{\partial V_{j i}\left(0, y_{d f}\right)}{\partial x_{j}\left(t-h_{j}\right)}\right] ;
\end{aligned}
$$

$j \in \bar{r} \cup\{0\}$ 


$$
\begin{gathered}
F_{e j}=\left[\frac{\partial F_{e j i}\left(x_{e n d}, y_{e n d}\right)}{\partial x_{j}\left(t-h_{j}\right)}\right] ; \\
V_{e j}=\left[\frac{\partial V_{e j i}\left(x_{e n d}, y_{e n d}\right)}{\partial x_{j}\left(t-h_{j}\right)}\right] ;
\end{gathered}
$$$$
j \in \bar{r} \cup\{0\},
$$

respectively, at the disease-free equilibrium point $z_{d f}=$ $\left(0^{T}, y_{d f}^{T}\right)^{T}$ and at the endemic one $z_{\text {end }}=\left(x_{\text {end }}^{T}, y_{\text {end }}^{T}\right)^{T}$ and the redefinitions:

$$
\begin{aligned}
& F=\sum_{i=0}^{r} F_{i}, \\
& V=\sum_{i=0}^{r} V_{i}
\end{aligned}
$$

The extended versions of Assumptions 1 and 2 are kept for the delayed epidemic models as follows.

Assumptions 4. $\sum_{j=0}^{r} F_{i j}\left(0, y\left(t-h_{j}\right)\right)=\sum_{j=0}^{r} V_{i j}\left(0, y\left(t-h_{j}\right)\right)=$ $0 ; \forall i \in \bar{n}=\{1,2, \ldots, n\}, F_{i j}\left(x\left(t-h_{j}\right), y\left(t-h_{j}\right)\right) \geq 0$, $V_{i j}\left(x\left(t-h_{j}\right), y\left(t-h_{j}\right)\right) \leq 0$ when $x_{i}\left(t-h_{j}\right)=0$, and $\sum_{i=1}^{n} \sum_{j=0}^{r} V_{i j}(x(t), y(t)) \geq 0$ for all $x(t), y(t) \geq 0$.

Assumptions 5. (a) $\left(-V_{0}\right) \in M_{E}^{n \times n}, V_{0}^{-1}>0, F_{i} \geq V_{i} ; \forall i \in \bar{r}$.

(b) The disease-free equilibrium point $z_{d f}=\left(0^{T}, y_{d f}^{T}\right)^{T}$ is unique and the noninfective subsystem $\dot{y}(t)=g(0, y(t))$ is locally asymptotically stable.

Example 11. A simple motivation of the model (24)-(26) subject to delays covers and generalizes classical models in the literature which have a physical interpretation. A well-known classical Kermack-McKendrick-based epidemic model in integral form, revisited in [27], is the subsequent one:

$$
\dot{S}(t)=S(t) \int_{0}^{\infty} \widetilde{A}(\tau) \dot{S}(t-\tau) d \tau
$$

where $S(t)$ denotes the spatial density of susceptibles, i.e., the number of individuals per area at time $t$ where $\widetilde{A}(\tau)$ is the expected infectivity of an individual which become infected $\tau$ unity of times ago. That model can be interpreted in differential form as the SI- epidemic model:

$$
\begin{aligned}
& \dot{S}(t)=-\beta S(t) I(t) ; \\
& \dot{I}(t)=\beta S(t) I(t)-\gamma I(t)
\end{aligned}
$$

by defining $I(t)=-\beta^{-1} \int_{-\infty}^{t} \widetilde{A}(t-\tau) \dot{S}(\tau) d \tau$, subject to the special infectivity $\widetilde{A}(\tau)=\beta e^{-\gamma \tau}$, where $\beta$ and $\gamma$ are the coefficient transmission rate and the infection rate. A more general differential epidemic model subject to time-delays becomes:

$$
\begin{aligned}
& \dot{S}(t)=-\beta \sum_{i=0}^{r} S\left(t-h_{i}\right) I\left(t-h_{i}\right) \\
& \dot{I}(t)=\beta \sum_{i=0}^{r} S\left(t-h_{i}\right) I\left(t-h_{i}\right)-\gamma \sum_{i=0}^{r} I\left(t-h_{i}\right)
\end{aligned}
$$

which corresponds to that in the integral form:

$$
\dot{S}(t)=\sum_{i=0}^{r} S\left(t-h_{i}\right) \int_{0}^{\infty} \widetilde{A}(\tau) \dot{S}\left(t-h_{i}-\tau\right) d \tau
$$

The physical interpretation relies on the arrival of several infectious groups at different time instants or to the combined contributions of several infective strains at different time instants both with the same coefficient rates.

Sufficient conditions for the stability of the linearized system independent of the delay sizes around the disease-free equilibrium point can be obtained for the cases when either $V_{0}$ or $V$ is a stability matrix. For this purpose, we first write the equations of the linearized system in two ways depending on the nonsingularity of $V_{0}$ or that of $V$ as follows.

Lemma 12. The following properties hold.

(i) The infective linearized subsystem obtained from (24)(25) around the disease-free equilibrium point is given by the subsequent dynamics:

$$
\dot{x}_{L}(t)=\sum_{i=0}^{r}\left(F_{i}-V_{i}\right) x_{L}\left(t-h_{i}\right)
$$

for given initial conditions $x_{L}(t)=x(t)=\varphi(t) ; \forall t \in[-h, 0]$, where $\varphi:[-h, 0] \longrightarrow \mathbf{R}^{n+m}$ is an absolutely continuous function with eventual finite jumps on a subset of $[-h, 0]$ of zero measure with $\varphi(0)=\left(x^{T}(0), y^{T}(0)\right)^{T}$.

(ii) If $V_{0}$ is nonsingular then (36) can be equivalently expressed as

$$
\begin{aligned}
\dot{x}_{L}(t)= & -V_{0}\left(I_{n}-V_{0}^{-1} F_{0}\right) x_{L}(t) \\
& +\sum_{i=1}^{r}\left(F_{i}-V_{i}\right) x_{L}\left(t-h_{i}\right) \\
= & \sum_{i=0}^{r}\left(F_{i}-V_{i}\right) x_{L}(t) \\
& +\sum_{i=1}^{r}\left(F_{i}-V_{i}\right)\left(x_{L}\left(t-h_{i}\right)-x_{L}(t)\right)
\end{aligned}
$$

(iii) If $V$ is nonsingular then (36) can be equivalently expressed as

$$
\begin{aligned}
\dot{x}_{L}(t) & \\
= & -\left(\sum_{i=0}^{r} V_{i}\right)\left(I_{n}-\left(\sum_{i=0}^{r} V_{i}\right)^{-1}\left(\sum_{i=0}^{r}\left(F_{i}\right)\right)\right) x_{L}(t) \\
& +\sum_{i=1}^{r}\left(F_{i}-V_{i}\right)\left(x_{L}\left(t-h_{i}\right)-x_{L}(t)\right)
\end{aligned}
$$




$$
\begin{aligned}
= & \left(\sum_{i=0}^{r}\left(F_{i}-V_{i}\right)\right) x_{L}(t) \\
& +\sum_{i=1}^{r}\left(F_{i}-V_{i}\right)\left(x_{L}\left(t-h_{i}\right)-x_{L}(t)\right)
\end{aligned}
$$

(iv) If $V_{0}$ and $V$ are nonsingular then the linearized system around the disease-free equilibrium point is described indistinctly by both (37) and (38) and also by

$$
\begin{gathered}
\dot{x}_{L}(t)=-V_{0}\left(I_{n}+V_{0}^{-1}\left(\sum_{i=1}^{r} V_{i}\right)\right) \\
\cdot\left(I_{n}-\left(V_{0}\left(I_{n}+V_{0}^{-1}\left(\sum_{i=1}^{r} V_{i}\right)\right)^{-1}\left(\sum_{i=0}^{r} F_{i}\right)\right)\right) \\
\cdot x_{L}(t)+\sum_{i=1}^{r}\left(F_{i}-V_{i}\right)\left(x_{L}\left(t-h_{i}\right)-x_{L}(t)\right)
\end{gathered}
$$

Proof. Eq. (36) is a linearized version of (24) around the disease-free equilibrium point. Eqs. (37) and (38) are direct from (36) if $V_{0}$ and $V$ are, respectively, nonsingular. Furthermore, if both matrices are nonsingular then $V^{-1}=\left(I_{n}+\right.$ $\left.V_{0}^{-1}\left(\sum_{i=1}^{r} V_{i}\right)\right)^{-1} V_{0}^{-1}$ and $\left(I_{n}+V_{0}^{-1}\left(\sum_{i=1}^{r} V_{i}\right)\right)^{-1}$ exists what leads to $(39)$.

Some basic results, given in Theorem 1 on positivity and stability for the delay-free linearized infective subsystem around the disease-free equilibrium point, for the case of multiple point delays follows.

Theorem 13. The following properties hold under Assumptions 4-5.

(i) The linearized epidemic model (24)-(25) around the disease-free equilibrium point has a nonnegative solution of the infective substate independent of the delays for any bounded admissible function of initial conditions $\varphi(t) \geq 0 ; \forall t \in[-h, 0]$ if and only if $\left(F_{0}-V_{0}\right) \in M_{E}^{n \times n}$ (equivalently, $\left(-V_{0}\right) \in M_{E}^{n \times n}$ and $\left.F_{0} \geq 0\right)$ and $F_{i} \geq V_{i} ; \forall i \in \bar{r}$. These conditions are also jointly necessary for the epidemic model (24)-(25) to have a nonnegative solution trajectory independent of the delays for any admissible function of initial conditions $\varphi(t) \geq 0 ; \forall t \in[-h, 0]$.

(ii) The linearized infective substate around the diseasefree equilibrium point has a nonnegative solution trajectory and it is globally exponentially stable independent of the delays for any admissible function of initial conditions $\varphi(t) \geq 0 ; \forall t \in[-h, 0]$ if the following conditions jointly hold: $\left(F_{0}-V_{0}\right) \in M_{E}^{n \times n}$ is a stability matrix (equivalently, it exists $\left(V_{0}-F_{0}\right)^{-1}>0$; equivalently $\left(-V_{0}\right) \in M_{E}^{n \times n}$, it exists $V_{0}^{-1}>0$, $F_{0} \geq 0$, and $\left.\rho_{0 \infty}=\rho\left(F_{0} V_{0}^{-1}\right)<1\right), F_{i} \geq V_{i} ; \forall i \in \bar{r}$, and

$$
\begin{aligned}
\bar{\rho}_{0 \infty} & =\left\|\left(s I_{n}+V_{0}-F_{0}\right)^{-1}\left(\sum_{i=1}^{r}\left(F_{i}-V_{i}\right) e^{-s h_{i}}\right)\right\|_{\infty} \\
& =\sup _{\omega \in \mathbf{R}_{0+}}\left\|\left(\mathbf{i} \omega I_{n}+V_{0}-F_{0}\right)^{-1}\left(\sum_{i=1}^{r}\left(F_{i}-V_{i}\right)\right)\right\|_{2}
\end{aligned}
$$

(iii) The linearized infective substate around the disease-free equilibrium point is positive and globally exponentially stable independent of the delays for any admissible function of initial conditions $\varphi(t) \geq 0 ; \forall t \in[-h, 0]$ if and only if the following conditions jointly hold: $\sum_{i=0}^{r}\left(F_{i}-V_{i}\right)$ is a stability matrix, $\left(F_{0}-V_{0}\right) \in M_{E}^{n \times n}$ (equivalently, $\left(-V_{0}\right) \in M_{E}^{n \times n}$ and $F_{0} \geq 0$ ), $F_{i} \geq V_{i} ; \forall i \in \bar{r}$, and the matrix $\left(\sum_{i=0}^{r}\left(F_{i}-V_{i}\right) e^{-s h_{i}}\right)$ has no nonzero imaginary eigenvalues.

(iv) The whole linearized model around the unique disease-free equilibrium point has a nonnegative state trajectory solution for any given admissible nonnegative function of initial conditions which converges asymptotically to the unique disease-free equilibrium point.

Proof. Note that the solution of the linearized infective substate around the disease-free equilibrium point is

$$
\begin{aligned}
x_{L}(t)= & e^{\left(F_{0}-V_{0}\right) t} x_{L}(0) \\
& +\sum_{i=1}^{r} \int_{0}^{t} e^{\left(F_{0}-V_{0}\right)(t-\tau)}\left(F_{i}-V_{i}\right) x_{L}\left(\tau-h_{i}\right) d \tau \\
= & e^{\left(F_{0}-V_{0}\right) t} x_{L}(0) \\
& +\sum_{i=1}^{r} \int_{-h_{i}}^{t-h_{i}} e^{\left(F_{0}-V_{0}\right)\left(t-h_{i}-\tau\right)}\left(F_{i}-V_{i}\right) x_{L}(\tau) d \tau \\
= & e^{\left(F_{0}-V_{0}\right) t} x_{L}(0) \\
& +\sum_{i=1}^{r} \int_{-h_{i}}^{0} e^{\left(F_{0}-V_{0}\right)\left(t-h_{i}-\tau\right)}\left(F_{i}-V_{i}\right) \varphi(\tau) d \tau \\
& +\sum_{i=1}^{r} \int_{0}^{t-h_{i}} e^{\left(F_{0}-V_{0}\right)\left(t-h_{i}-\tau\right)}\left(F_{i}-V_{i}\right) x_{L}(\tau) d \tau
\end{aligned}
$$

The sufficiency part is direct from (41) since if $\left(F_{0}-V_{0}\right) \epsilon$ $M_{E}^{n \times n}$ and $F_{i} \geq V_{i} ; \forall i \in \bar{r}$ then $x_{L}(t) \geq 0$ for any $t>0$ and any given $\varphi(t) \geq 0 ; \forall t \in[-h, 0]$. The necessity is proved by contradiction in two separate steps. Assume that $A_{0}=\left(A_{0_{i j}}\right)=\left(F_{0}-V_{0}\right) \notin M_{E}^{n \times n}$. Then, there is some pair $(i, k(\neq i)) \in \bar{n} \times \bar{n}$ such that $A_{0_{i k}}<0$. Now, take a function of initial conditions satisfying $\varphi_{k}(0)>0, \varphi_{i}(0)=0 ; \forall i(\neq k) \in \bar{n}$, and $\varphi(t)=0$ for $t \in[-h, 0)$. Thus, $\dot{x}_{L_{i}}(0)=-\left|A_{0_{i k}}\right| \varphi_{k}(0)<0$ and $x_{L_{i}}(0)=0$ so that $x_{L}\left(0^{+}\right)<0$ and the system is not positive. On the other hand, assume that there is some $i \in \bar{r}$ such that $V_{i}>F_{i}$ so that there is some pair $(j, k) \in \bar{n} \times \bar{n}$ such that $V_{i_{j k}}>F_{i_{j k}}$. Take initial conditions as follows: $\varphi(t)=0$ for $t \in\left[-h,-h_{i}\right) \cup\left(-h_{i}, 0\right], \varphi_{\ell}\left(-h_{i}\right)=0 ; \forall k, \ell(\neq k) \in \bar{n}$ and $\varphi_{k}\left(-h_{i}\right)>0$. Then, $\dot{x}_{L_{j}}(0)=-\left|F_{i_{j k}}-V_{i_{j k}}\right| \varphi_{k}\left(-h_{i}\right)<0$ and $x_{L_{j}}(0)=0$ so that $x_{L_{j}}\left(0^{+}\right)<0$ and the system is not positive. Therefore, $F_{i} \geq V_{i} ; \forall i \in \bar{r}$ and $\left(F_{0}-V_{0}\right) \in M_{E}^{n \times n}$ are necessary for positivity. The first part of Property (i) for the linearized system around the disease-free equilibrium point has been proved. The fact that the conditions are also necessary for the positivity of the whole nonlinear model are obvious in the guidelines of Theorem 1 (i) for the delayfree case since the positivity of the whole model requires that of its linearized version around the disease-free equilibrium 
point. This proves the second part of Property (i). Property (ii) contains conditions to guarantee the positivity (borrowed from Property (i)) and, furthermore, the global asymptotic stability is guaranteed as follows. Since the transfer matrix $\left(s I_{n}+V_{0}-F_{0}\right)^{-1}$ is stable, since $\left(F_{0}-V_{0}\right)$ is a stability matrix, that is $\operatorname{det}\left(s I_{n}+V_{0}-F_{0}\right) \neq 0$ for $\operatorname{Re} s \geq 0$, then condition (40) is equivalent to

$$
\begin{aligned}
& \operatorname{det}\left(\boldsymbol{\iota} \omega I_{n}+\sum_{i=0}^{r}\left(V_{i}-F_{i}\right) e^{-\imath \omega h_{i}}\right)=\operatorname{det}\left(\boldsymbol{\iota} \omega I_{n}+V_{0}\right. \\
& \left.-F_{0}\right) \operatorname{det}\left(I_{n}\right. \\
& \left.-\left(\boldsymbol{\iota} \omega I_{n}+\left(V_{0}-F_{0}\right)^{-1}\right)\left(\sum_{i=0}^{r}\left(V_{i}-F_{i}\right) e^{-\imath \omega h_{i}}\right)\right) \\
& \neq 0 ; \quad \forall \omega \in \mathbf{R}_{0+} .
\end{aligned}
$$

Thus, constraint (42) is a sufficient condition for global asymptotic stability. Since the linearized system is timeinvariant and globally asymptotically stable and since timeinvariant time-delay systems with constant point delays can also have a finite number of characteristic zeros to the right of any vertical line in the complex plane of abscissa greater than $-\infty$, then the solution is a function of time of exponential negative order so that one concludes that the proved global asymptotic stability is also exponential. Property (ii) has been proved. On the other hand, note that if the jointly condition that $\sum_{i=0}^{r}\left(F_{i}-V_{i}\right)$ is a stability matrix and the matrix $\left(\sum_{i=0}^{r}\left(F_{i}-\right.\right.$ $\left.\left.V_{i}\right) e^{-s h_{i}}\right)$ has no nonzero imaginary eigenvalues holds then the global asymptotic stability at exponential rate is also guaranteed since $\left(\sum_{i=0}^{r}\left(F_{i}-V_{i}\right) e^{-s h_{i}}\right)$ has no imaginary eigenvalues and it is a stability matrix in the delay-free case. Such a jointly condition is also necessary [28] for global asymptotic stability from the continuity of the characteristic zeros with respect to the parameters. Since the linearized system is time-invariant and globally asymptotically stable and since time-invariant time-delay systems with constant point delays can also have a finite number of characteristic zeros to the right of any vertical line in the complex plane of abscissa greater than $-\infty$, then the solution is a function of time of exponential negative order so that one concludes that the proved global asymptotic stability is also exponential. Property (iii) has been proved. Since Assumptions 4-5 hold, then both the linearized infective substate and the noninfective one around the unique disease-free equilibrium point have a nonnegative state trajectory solution which converges exponentially to the disease-free equilibrium point for any given admissible nonnegative function of initial conditions. Hence, Property (iv) is proved.

Taking into account (42) in the proof of Theorem 13, one gets the subsequent result.

Corollary 14. The linearized infective substate around the disease-free equilibrium point is positive and exponentially stable independent of the set of $r$ commensurate delays $h_{i}=i h_{1}$; $\forall i \in \bar{r}$ for any bounded admissible function of initial conditions $\varphi(t) \succeq 0 ; \forall t \in[-h, 0]$ if and only if $\sum_{i=0}^{r}\left(F_{i}-V_{i}\right)$ is a stability matrix, $\left(F_{0}-V_{0}\right) \in M_{E}^{n \times n}, F_{i} \succeq V_{i} ; \forall i \in \bar{r}$, and

$$
\operatorname{det}\left(\iota \omega I_{n}+\sum_{i=0}^{r}\left(V_{i}-F_{i}\right) z^{i}\right) \neq 0
$$

$\forall \omega \in \mathbf{R}_{0+}, \forall z \in C(0,1)$.

If the set $S=\left\{(\omega, z) \in \mathbf{R}_{0+} \times \partial C(0,1)\right\} \neq \varnothing$ then the infective substate around the disease-free equilibrium point is not exponentially stable for the sets of commensurate delays $S_{H}=\left\{h_{i}=-\ln z / \omega:(\omega, z) \in S\right\}$.

Taking into account the expression (36) to describe the linearized infective dynamics around the disease-free equilibrium point, it is possible to give an alternative sufficiencytype condition for global stability of the infective subsystem as the subsequent result reflects.

Corollary 15. The linearized infective substate around the disease-free equilibrium point is exponentially stable independent of the set of $r$ incommensurate delays $h_{i} ; \forall i \in \bar{r}$ if

$$
\begin{gathered}
\bar{\rho}_{00}=2\left\|\sum_{i=1}^{r}\left(F_{i}-V_{i}\right)\right\|_{2} \\
\cdot \sup _{\omega \in \mathbf{R}_{+}}\left\|\left(\mathbf{i} \omega I_{n}-\left(\sum_{i=1}^{r}\left(F_{i}-V_{i}\right)\right)\right)^{-1}\right\|_{2}<1
\end{gathered}
$$

provided that $\sum_{i=0}^{r}\left(F_{i}-V_{i}\right)$ is a stability matrix, which holds if and only if $\rho_{00}=\rho\left[\left(\sum_{i=0}^{r} F_{i}\right)\left(\sum_{i=0}^{r} V_{i}\right)^{-1}\right]<1$.

Proof. One gets the characteristic equation from (37) as follows:

$$
\begin{aligned}
& \operatorname{det}\left(s I_{n}-\left(\sum_{i=0}^{r}\left(F_{i}-V_{i}\right)\right)-\sum_{i=1}^{r}\left(F_{i}-V_{i}\right)\left(e^{-h_{i} s}-1\right)\right) \\
& =0 \text { for } s \in \mathbf{C}
\end{aligned}
$$

Since $\left(\sum_{i=0}^{r}\left(F_{i}-V_{i}\right)\right)$ is a stability matrix then $\operatorname{det}\left(s I_{n}-\right.$ $\left.\left(\sum_{i=0}^{r}\left(F_{i}-V_{i}\right)\right)\right) \neq 0$ for $s \in \mathbf{C}_{0+}$ so that

$$
\begin{aligned}
& \operatorname{det}\left(s I_{n}-\left(\sum_{i=0}^{r}\left(F_{i}-V_{i}\right)\right)\right) \operatorname{det}\left(I_{n}\right. \\
& -\left(s I_{n}-\left(\sum_{i=0}^{r}\left(F_{i}-V_{i}\right)\right)\right)^{-1} \\
& \left.\cdot\left(\sum_{i=1}^{r}\left(F_{i}-V_{i}\right)\left(e^{-h_{i} s}-1\right)\right)\right) \neq 0 ; \quad \forall s \in \mathbf{C}_{0+}
\end{aligned}
$$

provided that $\|\left(s I_{n}-\left(\sum_{i=0}^{r}\left(F_{i}-V_{i}\right)\right)\right)^{-1}\left(\sum_{i=1}^{r}\left(F_{i}-V_{i}\right)\left(e^{-h_{i} s}-\right.\right.$ $1)) \|_{\infty}<1$ which is guaranteed if $\bar{\rho}_{00}<1$. Since $\sum_{i=0}^{r}\left(F_{i}-V_{i}\right)$ is a stability matrix, the above test is fulfilled at the origin of the complex plane. Note also that $\sum_{i=0}^{r}\left(F_{i}-V_{i}\right)=\sum_{i=0}^{r}\left(-V_{i}\right)\left[I_{n}-\right.$ 
$\left.\sum_{i=0}^{r}\left(V_{i}\right)^{-1} \sum_{i=0}^{r} F_{i}\right]$ is a stability matrix if and only if $\rho_{00}<1$ since $\sum_{i=0}^{r}\left(-V_{i}\right)$ is a stability matrix. Therefore, a sufficient condition to achieve the global (exponential) asymptotic stability test independent of the delays is constraint (44) since $\sup _{\omega \in \mathbf{R}_{0+}}\left|e^{-\mathbf{i} h_{i} \omega}-1\right| \leq 2 ; \forall i \in \bar{r}$.

It follows from Corollary 15 and the "If Part" of Theorem 13 (iii) that since $\sum_{i=0}^{r}\left(F_{i}-V_{i}\right)$ is a stability matrix, constraint (44) guarantees that $\left(\sum_{i=0}^{r}\left(F_{i}-V_{i}\right) e^{-s h_{i}}\right)$ has no nonzero imaginary eigenvalues. Otherwise, the infective part of the linearized system around the disease-free equilibrium point could not be globally asymptotically stable.

Remark 16. The infective linearized system around the disease-free equilibrium point has two important particular cases which define auxiliary linearized systems which are useful for the local stability analysis independent of the delays, namely,

(a) auxiliary infective linearized delay-free system (AILDFS) describing the particular case $h_{i}=0 ; \forall i \in \bar{r}$ :

$$
\dot{x}_{L}(t)=\sum_{i=0}^{r}\left(F_{i}-V_{i}\right) x_{L}(t)
$$

for $t>0$ for admissible initial conditions $\varphi:[-h, 0] \longrightarrow \mathbf{R}_{0+}^{n}$ with $\varphi(0)=x(0)=x_{0}$ and $h=h_{r}$;

(b) auxiliary infective linearized delayed dynamics-free system (AILDDFS) describing the particular case $F_{i}-V_{i}=0$; $\forall i \in \bar{r}$ :

$$
\dot{x}_{L}(t)=\left(F_{0}-V_{0}\right) x_{L}(t)
$$

for $t>0$ for admissible initial conditions $\varphi:[-h, 0] \longrightarrow \mathbf{R}_{0+}^{n}$ with $\varphi(0)=x(0)=x_{0}$ and a given maximum delay $h=h_{r} \leq$ $+\infty$. This particular system can also be interpreted for the case of point initial conditions $\varphi(t)=0$ for $t \in[-\infty, 0)$ and $\varphi(0)=x(0)=x_{0}$ as defined under any amounts of delayed dynamics associated with infinity delays $h_{i}=+\infty ; \forall i \in \bar{r}$.

Note that the disease reproduction number $R_{0}\left(0, h_{1}\right.$, $\left.h_{2}, \ldots, h_{r}\right)$ is, in general, delay-dependent with particular values $R_{00}=R_{0}(\mathbf{0})$, with $\mathbf{0} \in \mathbf{R}^{n+1}$, which is the reproduction number of the AILDFS, and $R_{0 \infty}=R_{0}(0$, inf $)$, where inf $\epsilon$ $\mathbf{R}^{n}$ has all components equal to $+\infty$, which is the reproduction number of the AILDDFS. Define the maximum and minimum reproduction numbers for any given set of incommensurate delays:

$$
\begin{aligned}
& R_{M 0}=\max _{0 \leq h_{i} \leq \infty} R_{0}\left(0, h_{1}, h_{2}, \ldots, h_{r}\right) ; \\
& R_{m 0}=\min _{0 \leq h_{i} \leq \infty} R_{0}\left(0, h_{1}, h_{2}, \ldots, h_{r}\right)
\end{aligned}
$$

Note also that

$$
\begin{gathered}
R_{0}\left(0, h_{1}, h_{2}, \ldots, h_{r}\right) \in\left[R_{m 0}, R_{M 0}\right] \\
\forall h_{i} \in \mathbf{R}_{0+}, \forall i \in \bar{r}, \\
R_{00} \in\left[R_{m 0}, R_{M 0}\right], \\
R_{0 \infty} \in\left[R_{m 0}, R_{M 0}\right] .
\end{gathered}
$$

where $R_{00}=\max \left(\rho_{00}, \bar{\rho}_{00}\right), R_{0 \infty}=\max \left(\rho_{0 \infty}, \bar{\rho}_{0 \infty}\right)$. Note that $\rho_{00}$ and $\rho_{0 \infty}$ are the respective reproduction numbers of the AILDFS and the AILDDFS which are delay-free auxiliary linearized systems and the amounts $\bar{\rho}_{00}$ and $\bar{\rho}_{0 \infty}$ express the tolerance to stability independent of the delays of the linearized systems around the disease-free equilibrium point compatible with guaranteeing the stability independent of the delay sizes provided that those amounts are less than unity. Define $\bar{R}_{0}=\min \left(R_{00}, R_{0 \infty}\right)$ and note that $R_{m 0} \leq R_{0}\left(0, h_{1}\right.$, $\left.h_{2}, \ldots, h_{r}\right) \leq \bar{R}_{0} \leq R_{M 0} ; \forall h_{i} \in \mathbf{R}_{0+}, \forall i \in \bar{r}$.

Thus, the asymptotic stability independent of the delays of the linearized system around the disease-free equilibrium point holds if $R_{M 0} \leq 1$ and it is then guaranteed if $\bar{R}_{0} \leq 1$. However, the above constraint has only a partial information about the stability and attainability of the endemic equilibrium point for each particular combination of delays. For, instance, we can intuitively discuss the following cases.

(1) For a set of delays $h_{i} ; \forall i \in \bar{r}$, it holds that $R_{0}\left(0, h_{1}, h_{2}\right.$, $\left.\ldots, h_{r}\right) \leq 1<\bar{R}_{0} \leq R_{M 0}$. We get the conclusion that the disease-free equilibrium point is locally asymptotically stable and the endemic is unattainable for this value of $R_{0}(\cdot)$. Since $R_{M 0} \geq \bar{R}_{0}>1$, we can say that there are combinations of delays, all of them with the same amounts of delayed dynamics, for which the disease is endemic but it also holds that there exist some particular delays for which the disease asymptotically extinguishes. On the other hand, since $R_{M 0}>$ 1 , there are other sets of delays $\bar{h}_{i} ; \forall i \in \bar{r}$ for the same matrices of dynamics such that the disease-free equilibrium point is unstable and the endemic one is stable and attainable.

(2) For a set of delays $h_{i} ; \forall i \in \bar{r}$, it holds that $R_{0}\left(0, h_{1}, h_{2}\right.$, $\left.\ldots, h_{r}\right) \leq \bar{R}_{0}<1<R_{M 0}$. We get the conclusion that the disease-free equilibrium point is locally asymptotically stable and the endemic one is unattainable. However, the reproduction number range for which this holds exceeds the lower-bound given by $\bar{R}_{0}$. Furthermore, there are other sets of delays $\bar{h}_{i} ; \forall i \in \bar{r}$, under the same amounts of delayed dynamics, such that the disease-free equilibrium point is unstable and the endemic one is stable and attainable.

(3) In the inequalities of the above cases (a) and (b), if $\bar{R}_{0}$ is replaced with $R_{00}$ or $R_{0 \infty}$, we get similar conclusions.

The following three results discuss the stability and positivity independent of the delays of the linearized system around the disease-free equilibrium point if either $R_{00}$ (i.e., the reproduction number of the AILDFS) or $R_{0 \infty}$ (i.e., the reproduction number of the $A I L D D F S$ ) or both amounts are less than unity. In the first case, the complementary sufficienttype stability condition (44) of worst-case tolerance against delayed dynamics is also invoked. In the second case the complementary worst-case tolerance condition (40) replaces to $(44)$.

Corollary 17. If Assumption 5 holds then the following properties hold.

(i) If constraint (44) holds, then there exists a real number $\varepsilon=\varepsilon\left(h_{1}, h_{2}, \ldots, h_{r}\right) \in \mathbf{R}_{0+}$ dependent, in general, on the delays $h_{i} \geq 0$ for $i \in \bar{r}$, such that the reproduction number is given by $R_{0}\left(0, h_{1}, h_{2}, \ldots, h_{r}\right)=(1+\varepsilon) R_{00}-\varepsilon$, so that 
$\left(R_{00} \leq 1\right) \Longrightarrow\left(R_{0}\left(0, h_{1}, h_{2}, \ldots, h_{r}\right) \leq 1\right)$, and the implication also holds under strict inequalities, for any given set of delays $h_{i}$; $\forall i \in \bar{r}$, and then $R_{m 0} \leq R_{M 0} \leq 1$. Then, the infective substate of the linearized system around the disease-free equilibrium point is globally asymptotically stable independent of the delays, with a nonnegative state trajectory solution for any given admissible nonnegative function of initial conditions, if $R_{00}<1$.

(ii) The AILDFS is globally asymptotically stable around the disease-free equilibrium point, with a nonnegative state trajectory solution for any given admissible nonnegative function of initial conditions, if and only if $\rho_{00} \leq 1$. If $\rho_{00}<1$ then, furthermore, the endemic equilibrium point is unattainable. If $\rho_{00}=1$ then the disease-free and the endemic equilibrium points coincide. If $\rho_{00}>1$ then the disease-free equilibrium point is unstable.

Proof. The positivity and stability are guaranteed for the linearized infective substate from Theorem 13 (ii) and Lemma 12 (ii). Now assume that $\rho_{00} \leq 1$ and (44) holds, that is, if $\bar{\rho}_{00}<1$, both combined guarantee that $R_{00}<1$. Note that the critical stability of the infective linearized system around the diseasefree equilibrium point is given by $\rho_{00}=1$. It is now proved that $\left(R_{00} \leq 1\right) \Longrightarrow\left(R_{0}\left(0, h_{1}, h_{2}, \ldots, h_{r}\right) \leq 1\right)$ for any set of incommensurate delays $h_{i} ; \forall i \in \bar{r}$ if (44) holds. Assume, on the contrary, that $\left(R_{00} \leq 1\right) \Longrightarrow\left(R_{0}\left(0, h_{1}, h_{2}, \ldots, h_{r}\right)>\right.$ 1) for some set of delays $h_{i} ; \forall i \in \bar{r}$, so that $R_{M 0}>1$. Then, the linearized infective substate around the disease-free equilibrium point is unstable for at least one set of delays, a contradiction to Theorem 13 (ii) from the joint conditions $\rho_{00}<1$ and (44). Hence, $\left(R_{00} \leq 1\right) \Longrightarrow\left(R_{0}\left(0, h_{1}, h_{2}, \ldots, h_{r}\right) \leq\right.$ 1). Since both $R_{0}\left(0, h_{1}, h_{2}, \ldots, h_{r}\right)$ and $R_{00}$ are positive real constants which depend on the model parameterization then

$$
\begin{aligned}
R_{0}\left(0, h_{1}, h_{2}, \ldots, h_{r}\right) & =(1+\varepsilon) R_{00}-\varepsilon \\
& =\left(R_{00}-1\right) \varepsilon+R_{00}
\end{aligned}
$$

for some $\varepsilon=\varepsilon\left(h_{1}, h_{2}, \ldots, h_{r}\right) \in \mathbf{R}_{0+}$ for each set of delays $h_{i} \geq 0 ; \forall i \in \bar{r}$. The nonnegativity of any solution under any nonnegative admissible initial conditions follows from Assumption 5. Property (i) has been proved. Property (ii) follows since if Assumption 5 holds, then the AILDFS is globally asymptotically stable if and only if $\rho_{00} \leq 1$ since $\rho_{00}$ is the reproduction number of the AILDFS and any trajectory solution is nonnegative under nonnegative initial conditions. The other assertions in Property (ii) follow from Theorem 4 (ii) since the AILDFS is a delay-free system.

Corollary 18. Under Assumption 5, if $V=\sum_{i=0}^{r} V_{i}$ is nonsingular and (40) holds then the following properties hold.

(i) $R_{0}\left(0, h_{1}, h_{2}, \ldots, h_{r}\right)=\left(1+\varepsilon_{1}\right) R_{0 \infty}-\varepsilon_{1}$ for any given set of delays $h_{i} \geq 0$ for $i \in \bar{r}$ and some $\varepsilon_{1}=\varepsilon_{1}\left(h_{1}, h_{2}, \ldots, h_{r}\right) \in \mathbf{R}_{0+}$. Thus, the infective substate of the linearized system around the disease-free equilibrium point is globally asymptotically stable independent of the delays with a nonnegative state trajectory solution for any given admissible nonnegative function of initial conditions if $\rho_{0 \infty} \leq 1$ and (40) holds, that is, if $\bar{\rho}_{0 \infty}<1$.

(ii) the AILDDFS is globally asymptotically stable around the disease-free equilibrium point, with a nonnegative state trajectory solution for any given admissible nonnegative function of initial conditions, if and only if $\rho_{0 \infty}<1$. If $\rho_{0 \infty}<1$ then the endemic equilibrium point of the AILDDFS is unattainable. If $\rho_{0 \infty}=1$ then the disease-free and the endemic equilibrium points coincide. If $\rho_{0 \infty}>1$ then the disease-free equilibrium point is unstable.

Proof. It follows under a similar reasoning as that in the proof of Corollary 17 by using Lemma 12 (iii).

Corollary 19. If Assumption 5 holds, $V$ is nonsingular and (40) and (44) jointly hold, then $R_{0}\left(0, h_{1}, h_{2}, \ldots, h_{r}\right)=(1+$ $\left.\varepsilon_{2}\right) \bar{\rho}-\varepsilon_{2}$ for any given set of delays $h_{i} \geq 0$ for $i \in \bar{r}$ and some $\varepsilon_{2}=\varepsilon_{2}\left(h_{1}, h_{2}, \ldots, h_{r}\right) \in \mathbf{R}_{0+}$, where $\bar{R}_{0}=\max \left(R_{00}, R_{0 \infty}\right)<1$, and then the infective substate of the linearized system around the disease-free equilibrium point has a globally asymptotically stable nonnegative state trajectory solution independent of the delays for any given nonnegative admissible function of initial conditions.

Proof. It follows under a similar reasoning as that in the proof of Corollary 17 by taking into account Corollary 18.

Remark 20. If Assumption 5 holds, and $V=\sum_{i=0}^{r} V_{i}$ and $V^{\prime}=\sum_{i=1}^{r} V_{i}=V-V_{0}$ are nonsingular then $\bar{\rho}_{1}=$ $\max \left(\rho\left(F_{0} V_{0}^{-1}\right), \rho\left(\left(\sum_{i=0}^{r} F_{i}\right)\left(\sum_{i=0}^{r} V_{i}\right)^{-1}\right)\right)=\bar{\rho} \leq 1$ and then the condition $R_{0}\left(0, h_{1}, h_{2}, \ldots, h_{r}\right) \leq \bar{\rho}_{1}=\bar{\rho}$ for some set of delays $h_{i} \geq 0 ; \forall i \in \bar{r}$ if $\bar{\rho} \leq 1$ if both (40) and (44) hold included in Corollary 19. Thus, the linearized description of Lemma 12 (iv) does not add any new information to reformulate alternative conditions to those of Corollary 19.

Remark 21. Note that, since Theorem 13 (ii) gives sufficient (while not necessary) stability conditions independent of the delays, it cannot be proved in Corollary 17 that $\left(R_{00}>\right.$ $1) \Longrightarrow\left(R_{0}\left(0, h_{1}, h_{2}, \ldots, h_{r}\right)>1\right)$ for some set of delays $h_{i} \geq 0 ; \forall i \in \bar{r}$ to conclude instability. That is, $R_{00} \leq 1$ guarantees stability since $R_{0}\left(0, h_{1}, h_{2}, \ldots, h_{r}\right) \leq 1$ but the converse is not true, in general. In fact, if $R_{00}>1$ then $R_{0}\left(0, h_{1}, h_{2}, \ldots, h_{r}\right)>R_{00}$ since $\varepsilon\left(R_{00}-1\right)>0$ so that $\rho_{00}$ is a lower-bound of the maximum reproduction number for any set of admissible delays. Similar considerations can be said related to Corollaries 18 and 19 which do not provide necessary conditions either, in general, having in mind Theorem 13. So, $\rho_{0 \infty}$ is also a lower-bound of the maximum reproduction number for any set of admissible delays.

Remark 22. A case of interest might be when $F_{i}=\alpha_{i} F_{0}$; $i \in \bar{r} \cup\{0\}$ with $\alpha_{0}=1$. Then, $\rho\left(\left(\sum_{i=0}^{r} F_{i}\right)\left(\sum_{i=0}^{r} V_{i}\right)^{-1}\right)=$ $\left(1+\sum_{i=1}^{r} \alpha_{i}\right) \rho\left(F_{0}\left(\sum_{i=0}^{r} V_{i}\right)^{-1}\right)$ in Corollaries 18 and 19. If $0<$ $\alpha_{i+1} \leq \alpha_{i}$ (respectively, $\alpha_{i+1}>\alpha_{i}$ ) for $i \in \overline{r-1} \cup\{0\}$ then the delays lose their influence, or, at least, such an influence does not increase (respectively, increases) in the transmission of the disease as the delay sizes increase. So, $\rho\left(\left(\sum_{i=0}^{r} F_{i}\right)\left(\sum_{i=0}^{r} V_{i}\right)^{-1}\right) \leq(r+1) \rho\left(F_{0}\left(\sum_{i=0}^{r} V_{i}\right)^{-1}\right)$, respectively, $\rho\left(\left(\sum_{i=0}^{r} F_{i}\right)\left(\sum_{i=0}^{r} V_{i}\right)^{-1}\right) \geq(r+1) \rho\left(F_{0}\left(\sum_{i=0}^{r} V_{i}\right)^{-1}\right)$.

Example 23. Consider the subsequent linearized model around the disease-free equilibrium point:

$$
\dot{x}_{L}(t)=\sum_{i=0}^{1}\left(F_{i}-V_{i}\right) x_{L}\left(t-h_{i}\right)
$$


for any delay $h \geq 0$. The nonnegativity of the solutions of the linearized system holds with $\left(-V_{0}\right) \in M_{E}^{n \times n}$ and $F_{0} \geq 0$ (AILDDFS-positivity, that is, $\left(F_{0}-V_{0}\right) \in M_{E}^{n \times n}$ ) and $F_{1} \geq V_{1}$ (these three joint conditions implying AILDFS-positivity, that is, $\left.\sum_{i=0}^{1}\left(F_{i}-V_{i}\right) \in M_{E}^{n \times n}\right)$. If furthermore, the nonlinear contribution to the infective dynamics of the whole epidemic model fulfills $-f(\widehat{x}(t), \widehat{y}(t)) \geq 0 ; \forall t \in \mathbf{R}_{0+}$, independent of $h$, then the whole solution trajectory is nonnegative for all time independent of $h$. The relevant amounts to guarantee the asymptotic stability of the auxiliary delay-free systems around the disease-free equilibrium point and to keep it under a certain tolerance to the delayed dynamics amount (specified by the matrix $\left(F_{1}-V_{1}\right)$ ) independently of the delay $h$, via Theorem 13 and Corollaries 15 and 17, are

$$
\begin{aligned}
\rho_{0 \infty} & =\rho\left(F_{0} V_{0}^{-1}\right) ; \\
\bar{\rho}_{0 \infty} & =\sup _{\omega \in \mathbf{R}_{0+}}\left\|\left(\mathbf{i} \omega I_{n}+V_{0}-F_{0}\right)^{-1}\left(F_{1}-V_{1}\right)\right\|_{2} ; \\
\rho_{00} & =\rho\left[\left(F_{0}+F_{1}\right)\left(V_{0}+V_{1}\right)^{-1}\right] ; \\
\bar{\rho}_{00} & =2\left\|F_{1}-V_{1}\right\|_{2} \sup _{\omega \in \mathbf{R}_{+}}\left\|\left(\mathbf{i} \omega I_{n}-\left(F_{1}-V_{1}\right)\right)^{-1}\right\|_{2} ; \\
\bar{R}_{0} & =\min \left(R_{00}, R_{0 \infty}\right) ; \\
R_{00} & =\max \left(\rho_{00}, \bar{\rho}_{00}\right) ; \\
R_{0 \infty} & =\max \left(\rho_{0 \infty}, \bar{\rho}_{0 \infty}\right) .
\end{aligned}
$$

Note that

(1) $\rho_{0 \infty} \leq 1$ if and only if the AILDDFS is locally asymptotically stable. Since the endemic equilibrium point is not attainable if $\rho_{0 \infty}<1$ and it is confluent with the disease-free one if $\rho_{0 \infty}=1$, the condition also implies that the AILDDFS is globally asymptotically stable.

(2) $\rho_{0 \infty}>1$ implies that the disease-free equilibrium point is unstable and the endemic one is stable and attainable.

(3) $\rho_{00} \leq 1$ if and only if the AILDFS is locally asymptotically stable. Since the endemic equilibrium point is not attainable if $\rho_{00}<1$ and it is confluent with the diseasefree one if $\rho_{00}=1$, the condition also implies that the AILDFS is globally asymptotically stable.

(4) $\rho_{00}>1$ implies that the disease-free equilibrium point is unstable and the endemic one is stable and attainable.

(5) If $R_{0 \infty}<1$, or if $R_{00}<1$, then all the disease-free equilibrium points (which satisfy the respective conditions $\bar{\rho}_{0 \infty}<1$ or $\left.\bar{\rho}_{00} \leq 1\right)$ for any value of $h \geq 0$ are globally asymptotically stable. In particular, $F_{0}-V_{0}$ and $F_{1}-V_{1}$ are such that either $\bar{\rho}_{00} \leq 1$ or $\bar{\rho}_{0 \infty} \leq 1$ so that the corresponding auxiliary delay-free system around the disease-free equilibrium point is globally asymptotically stable. Under those respective conditions, all the resulting endemic equilibrium points are unattainable. They are attainable and stable if $R_{0 \infty} \geq 1$ or if $R_{00} \geq 1$.

The following result relies on sufficiency-type conditions of global asymptotic stability independent of the delays of the linearized infective substate around the disease-free equilibrium point including the case when the transmission matrices for the various delays are all identical.
Theorem 24. The following properties hold:

(i) Assume that $\left(F_{0}-V_{0}\right)$ and $\sum_{i=0}^{r}\left(F_{i}-V_{i}\right)$ are stability matrices, and that

$$
\begin{aligned}
& \rho\left[\left(I_{n}, I_{n}, \stackrel{r}{\cdot}, I_{n}\right)^{T}\left(\mathbf{i} \omega I_{n}-F_{0}+V_{0}\right)^{-1}\right. \\
& \left.\cdot\left(F_{1}-V_{1}, F_{2}-V_{2}, \ldots, F_{r}-V_{r}\right)\right]<1 ; \quad \forall \omega \in \mathbf{R}_{0+} \cdot
\end{aligned}
$$

Then, the infective linearized subsystem around the disease-free equilibrium point is globally asymptotically stable independent of the delays.

(ii) If $F_{i}=F_{0}>0 ; \forall i \in \bar{r},\left(-V_{0}\right)$ and $(-V)=-\sum_{i=0}^{r} V_{i}$ are stability matrices, $\rho\left(F_{0} V^{-1}\right)<1 /(r+1)$, and

$$
\begin{aligned}
& \rho\left[\left(I_{n}, I_{n}, \stackrel{r}{\cdot}, I_{n}\right)^{T}\left(\mathbf{i} \omega I_{n}-F_{0}+V_{0}\right)^{-1}\right. \\
& \left.\cdot\left(F_{0}-V_{1}, F_{0}-V_{2}, \ldots, F_{0}-V_{r}\right)\right]<1 ; \quad \forall \omega \in \mathbf{R}_{0+} \cdot
\end{aligned}
$$

Then the infective linearized subsystem is globally asymptotically stable independent of the delays.

Proof. If $\left(F_{0}-V_{0}\right)$ and $\sum_{i=0}^{r}\left(F_{i}-V_{i}\right)$ are stability matrices then the linearized subsystem is globally asymptotically stable for infinity and zero delays and the property also holds independent of the sizes of the delays [18] if (54) holds. Property (i) has been proved. Property (ii) follows in the same way when $F_{i}=F_{0}>0 ; \forall i \in \bar{r}$ since $\rho\left(F_{0} V^{-1}\right)<$ $1 /(r+1)$ guarantees that $(-V)$ is a stability matrix if $\left(-V_{0}\right)$ is a stability matrix and (55) is identical to (54) if $F_{i}=F_{0}>0$; $\forall i \in \bar{r}$.

Remark 25. Condition 2 of Theorem 24 holds if $\left(-V_{0}\right)$ is a nonsingular stability matrix (it suffices, for instance, that $\left(-V_{0}\right) \in M_{E}^{n \times n}$ and it exists $V_{0}^{-1}>0$, the second condition holds if and only if the Metzler matrix $\left(-V_{0}\right)$ is a stability matrix) and $\rho\left(V_{0}^{-1}\left(\sum_{i=1}^{r} V_{i}\right)\right)<1$ since such a condition implies that $(-V)$ is a stability matrix provided that $\left(-V_{0}\right)$ is also a stability matrix.

A related result to Theorem 4 for the case of presence of delays adopts the subsequent form.

Theorem 26. Let $g: \mathbf{R}^{n+m} \longrightarrow \mathbf{R}^{m}$ be everywhere continuously differentiable with a Jacobian matrix: $J_{g, z}\left(z_{0}\right)=$ $\left[J_{g, x}\left(z_{0}\right) \mid J_{g, y}\left(z_{0}\right)\right]$ at any $z_{0}=\left(x_{0}^{T}, y_{0}^{T}\right)^{T} \in \mathbf{R}^{n+m}$ with $x_{0} \in$ $\mathbf{R}^{n}$ and $y_{0} \in \mathbf{R}^{m}$, where $z=\left(x^{T}, y^{T}\right)^{T} \in \mathbf{R}^{n+m}, x \in \mathbf{R}^{n}$, $y \in \mathbf{R}^{m}$. Assume that the $m \times m$ matrix $J_{g, y}\left(z_{0}\right)$ is nonsingular at any equilibrium point $z_{0}$ of (24)-(26). Then, the following properties hold.

(i) There exists an open set $U$ of $\mathbf{R}^{n}$ containing $x_{0}$ such that there exists a unique continuously differentiable function $h: U \longrightarrow \mathbf{R}^{m}$ such that $y_{0}=h\left(x_{0}\right)$ and $f(x, h(x))=0$; $\forall x \in U$. Also, any equilibrium point $z_{0}=\left(x_{0}^{T}, y_{0}^{T}\right)^{T}$ can be 
expressed being dependent only on the infective substate, i.e., $z_{0}=\left(x_{0}^{T}, h^{T}\left(x_{0}\right)\right)^{T}$, while satisfying

$$
\begin{aligned}
& y_{0}=h\left(x_{0}\right) \\
& x_{0}=\left[\sum_{i=0}^{r}\left(F_{i}\left(h\left(x_{0}\right)\right)-V_{i}\left(x_{0}\right)\right)\right]^{-1} f\left(x_{0}, h\left(x_{0}\right)\right)
\end{aligned}
$$

provided that $\left[\sum_{i=0}^{r}\left(F_{i}\left(h\left(x_{0}\right)\right)-V_{i}\left(x_{0}\right)\right)\right]^{-1}$ is nonsingular at any equilibrium point $z_{0}=\left(x_{0}^{T}, h^{T}\left(x_{0}\right)\right)^{T}$, where $F_{i}(h(0))=F_{i}$ and $V_{i}\left(z_{0}\right)=V_{i} ; \forall i \in \bar{r} \cup\{0\}$ if $z_{0}=z_{d f}=\left(0^{T}, h^{T}(0)\right)^{T}$ is the disease-free equilibrium point and $F_{i}\left(h\left(x_{\text {end }}\right)\right)=F_{i e}$ and $V_{i}\left(x_{\text {end }}\right)=V_{i e} ; \forall i \in \bar{r} \cup\{0\}$ if $z_{0}=z_{\text {end }}=\left(x_{\text {end }}^{T}, h^{T}\left(x_{\text {end }}\right)\right)^{T}$ is the endemic equilibrium point.

(ii) Assume, furthermore, that $\left(-\sum_{i=0}^{r} V_{i}\right) \in M_{E}^{n \times n}$ is nonsingular with $F_{0} \geq 0$. Then, (a) the disease-free equilibrium point exists being defined by $z_{d f}=\left(0^{T}, h^{T}(0)\right)^{T}$, it is unique and locally asymptotically stable independent of the delays if $R_{00}<1$ and

(b) the endemic equilibrium point exists being defined by $z_{\text {end }}=\left(x_{\text {end }}^{T}, y_{\text {end }}^{T}\right)^{T}=\left(x_{\text {end }}^{T}, h^{T}\left(x_{\text {end }}\right)\right)^{T}$ while it satisfies

$$
\begin{aligned}
& y_{\text {end }}=h\left(x_{\text {end }}\right) ; \\
& x_{\text {end }}=\left(F_{e}-V_{e}\right)^{-1} f\left(x_{\text {end }}, h\left(x_{\text {end }}\right)\right) .
\end{aligned}
$$

It is reachable independent of the delays if $R_{00} \geq 1$ and $f\left(x_{\text {end }}, h\left(x_{\text {end }}\right)\right) \preceq f\left(x_{d f}, h\left(x_{d f}\right)\right)$ while it is unreachable, in the sense that $z_{\text {end }} \notin \mathbf{R}_{0+}^{n+m}$, if $R_{00}<1$ supposing that $f\left(x_{\text {end }}, h\left(x_{\text {end }}\right)\right)>f\left(x_{d f}, h\left(x_{d f}\right)\right)$ if $z_{\text {end }} \neq z_{d f}$.

Proof. Since $J_{g, z}\left(x_{0}, y_{0}\right)$ is nonsingular, there exists an open set $U$ of $\mathbf{R}^{n}$ containing $x_{0}$ such that there exists a unique continuously differentiable function $h: U \longrightarrow \mathbf{R}^{m}$ such that $y_{0}=h\left(x_{0}\right)$ and $f(x, h(x))=0 ; \forall x \in U$. Let $z_{0}$ be either the disease-free equilibrium point $z_{0}=z_{d f}=\left(0^{T}, y_{0}^{T}\right)^{T}$ or the endemic one $z_{0}=z_{\text {end }}=\left(x_{\text {end }}^{T}, y_{\text {end }}^{T}\right)^{T}$. Property (i) follows if $\left[\sum_{i=0}^{r}\left(F_{i}\left(h\left(x_{0}\right)\right)-V_{i}\left(x_{0}\right)\right)\right]^{-1}$ exists for any equilibrium point.

To prove Property (ii), note that $(-V) \in M_{E}^{n \times n}$ is nonsingular with $F \geq 0$. Then, the disease-free equilibrium point exists being defined by $z_{d f}=\left(0^{T}, h^{T}(0)\right)^{T}$, it is unique since $\mathrm{h}(\cdot)$ is unique, given by $x_{d f}=(F-V)^{-1} f(0, h(0))=$ 0 and it is locally asymptotically stable if $R_{00}<1$ since then $(F-V) \in M_{E}^{n \times n}$ is a stability matrix, equivalently $(V-F)^{-1}>0$ so that the linearized system around the disease-free equilibrium point is locally exponentially (then asymptotically ) stable. On the other hand, it is unstable if $R_{00}>1$ [Theorem 1 (iii)-(iv)]. The part (a) of Property (ii) has been proved. Now let $z_{0}=z_{\text {end }}=\left(x_{\text {end }}^{T}, y_{\text {end }}^{T}\right)^{T}$ be the endemic equilibrium point. Note that it is also unique, since $f$ and $h$ are everywhere continuous. Note that the following cases can occur.

(a) If $R_{00} \geq 1$, note that

$$
\begin{aligned}
x_{\text {end }} & =\left(V_{e}-F_{e}\right)^{-1}\left(-f\left(x_{\text {end }}, h\left(x_{\text {end }}\right)\right)\right) \\
& \geq(V-F)^{-1}\left(-f\left(x_{d f}, h\left(x_{d f}\right)\right)\right) \geq 0
\end{aligned}
$$

since $f\left(x_{\text {end }}, h\left(x_{\text {end }}\right)\right) \preceq 0$ (being a strict inequality if and only if $\left.x_{\text {end }} \neq x_{d f}=0\right)$ and $(F-V) \in M_{E}^{n \times n}$ is stable, then it is nonsingular.

(b) If $R_{00}<1$ then $(V-F)^{-1}>0$, since $(F-V) \in M_{E}^{n \times n}$, and $F_{e}>F$. Therefore, $\left(I_{n}+(V-F)^{-1}\left(F_{e}-F\right)\right)>0$ and $\rho\left[(V-F)^{-1}\left(F_{e}-F\right)\right]>0$ then $\left(I_{n}+\left(I_{n}-V^{-1} F\right)^{-1} V^{-1}\left(F_{e}-F\right)\right)^{-1}$ exists, and

$$
\begin{aligned}
& \left(V_{e}-F_{e}\right)^{-1}=\left(I_{n}+(V-F)^{-1}\left(F_{e}-F\right)\right)^{-1}(V-F)^{-1} \\
& \quad=\left(I_{n}+\left(I_{n}-V^{-1} F\right)^{-1} V^{-1}\left(F_{e}-F\right)\right)^{-1}(V-F)^{-1} \\
& \quad \prec(V-F)^{-1}
\end{aligned}
$$

Note that $x_{\text {end }}$ is unreachable if $x_{\text {end }} \neq x_{d f}=0$ since $-f\left(x_{\text {end }}, h\left(x_{\text {end }}\right)\right)>-f\left(x_{d f}, h\left(x_{d f}\right)\right)=0$, and

$$
\begin{aligned}
x_{\text {end }}= & \left(V_{e}-F_{e}\right)^{-1}\left(-f\left(x_{\text {end }}, h\left(x_{\text {end }}\right)\right)\right) \\
\prec & \left(I_{n}+\left(I_{n}-V^{-1} F\right)^{-1} V^{-1}\left(F_{e}-F\right)\right)^{-1} \\
& \times(V-F)^{-1}\left(-f\left(x_{d f}, h\left(x_{d f}\right)\right)\right) \\
= & \left(I_{n}+\left(I_{n}-V^{-1} F\right)^{-1} V^{-1}\left(F_{e}-F\right)\right)^{-1} x_{d f}=0 .
\end{aligned}
$$

Then, $x_{\text {end }} \preceq 0$. But, for the case, $x_{\text {end }}=0$, the endemic equilibrium point is coincident with the disease-free one, that is, $x_{\text {end }}=x_{d f}=0$ and $R_{00}=1$ while, for $R_{00}<1, x_{\text {end }} \prec$ 0 , hence a contradiction to its reachability follows since the infective components of the endemic equilibrium point are never allocated in the open first orthant of $\mathbf{R}^{n}$. Property (ii) has been proved.

The above result can be reformulated directly under the replacements $R_{00} \longrightarrow R_{0 \infty}$ and $R_{00} \longrightarrow \bar{R}_{0}$. Note that if $R_{00} \leq$ 1 or $R_{0 \infty} \leq 1$ or $\bar{R}_{0} \leq 1$, one concludes that the disease-free is guaranteed to be locally asymptotically stable independent of the delays. It can also be guaranteed that the endemic equilibrium point is attainable and stable independent of the delays if $\bar{R}_{00} \geq 1$, or $R_{0 \infty} \geq 1$, or $\bar{R}_{0} \geq 1$. However, combinations of delays with identical amounts of associated delayed dynamics can exist with reproduction numbers less than $R_{00}$, respectively, $R_{0 \infty}$, or, respectively, $\bar{R}_{0}$ so that the disease-free equilibrium point is unstable and the endemic one stable and attainable. From Theorem 13, and based on the previous results of Theorem 10 for the delay-free case, the following result holds on positivity and global asymptotically stability of the system subject to delays.

Theorem 27. Consider the epidemic model (27)-(29) under Assumptions 4-5 and, furthermore, assume that

(1) any of the sets of conditions of either Theorem 13 (ii), or Theorem 13 (iii), or Corollary 18(ii), or Corollary 19 hold,

(2) there are a unique disease-free equilibrium point and a unique endemic equilibrium point,

(3) $g_{i}(\widehat{z}(t)) \geq 0$ in (28) if $y_{i}(t)=0$ for any $i \in \bar{m}$ and $t \in \mathbf{R}_{0+}$, 
(4) $f_{i}(\widehat{z}(t)) \leq e_{i}^{T}(F-V) x(t)$ if $x_{i}\left(t-h_{j}\right)=0$ for any $i \epsilon$ $\bar{n} ; \forall j \in \bar{r} \cup\{0\}$ and $t \in \mathbf{R}_{0+}$ (it suffices that $f_{i}(\widehat{z}(t)) \leq 0$ if $\left.x_{i}\left(t-h_{j}\right)=0\right)$,

(5) the epidemic model is subject to any bounded absolutely continuous function of initial conditions $\varphi:[-h, 0] \longrightarrow$ $\mathbf{R}_{0+}^{n+m}$ with eventual finite jumps on a subset of $[-h, 0]$ of zero measure, where $\varphi(0)=\left(x^{T}(0), y^{T}(0)\right)^{T}$, such that the resulting function of initial conditions $\bar{\varphi}:[-h, 0] \longrightarrow \mathbf{R}_{0+}^{n+m}$.

(i) Then, any trajectory solution is nonnegative and uniformly bounded independent of the delays while it is globally convergent to the disease-free equilibrium point if $\bar{R}_{0}<1$, which is also a globally asymptotically stable attractor.

If furthermore, the following condition holds:

(6) if $\bar{R}_{0}>1$ then the total population of (27)-(29), i.e., the sum of all the subpopulations, is uniformly bounded independent of the delays for all time.

(ii) Then, provided that $f(\widehat{x}(t), \hat{y}(t))$ and $g(\widehat{x}(t), \hat{y}(t))$ are uniformly continuous in the first closed orthant of $\mathbf{R}^{n+m}$, all the trajectory solutions are bounded and nonnegative for all time while they converge asymptotically to the endemic equilibrium point which is also the unique asymptotically stable attractor.

Proof. If $\bar{R}_{0}<1$ then conditions (1)-(5) with Assumptions 4-5 guarantee that the linearized model around the diseasefree equilibrium point is locally asymptotically stable and all the trajectories within the open first orthant of $\mathbf{R}^{n+m}$ remain within it for all time. Since it is the unique attainable equilibrium the local asymptotic stability is also global (that is, for any bounded function of initial conditions within the first orthant) and asymptotic. Since all the subpopulations of both the infective and noninfective compartments are bounded and nonnegative for all time, the total population is also nonnegative and bounded through time. Property (i) has been proved. On the other hand, if condition (6) holds and $\bar{R}_{0}>1$ then the disease-free equilibrium point is unstable and the endemic one is attainable. Assume the two subsequent cases potentially to occur.

Case $a$. The endemic equilibrium point is globally asymptotically stable independent of the delays. Property (ii) is proved directly.

Case $b$. The endemic equilibrium point is unstable independent of the delays. Since the total population is bounded by hypothesis and all the subpopulations are nonnegative for all time, all the subpopulations are bounded for all time and a bounded limit cycle should surround the endemic point to attract any trajectories in the first orthant from the admissible Poincaré-type combinations of allowed stable/instable combinations of configurations of singular points and limit cycles. Rewrite compactly (27) to (29) in the form $\dot{z}(t)=$ $\widehat{f}(\widehat{x}(t), \hat{y}(t))$ by defining $\hat{f}: \mathbf{R}_{0+} \times \mathbf{R}^{n} \times \mathbf{R}^{m} \longrightarrow \mathbf{R}^{n+m}$ from the linearized infective dynamics and the functions $f: \mathbf{R}_{0+} \times \mathbf{R}^{n+m} \longrightarrow \mathbf{R}^{n}$ and $g: \mathbf{R}_{0+} \times \mathbf{R}^{n+m} \longrightarrow \mathbf{R}^{m}$. Assume that $\int_{0}^{\infty} \widehat{f}(\widehat{x}(\tau), \hat{y}(\tau)) d \tau= \pm \infty$. Then $z(t) \longrightarrow \pm \infty$ as $t \longrightarrow \infty$ which is not compatible with the nonnegativity of the solution trajectories within the first orthant and their boundenness. Therefore, $-\infty<\int_{0}^{\infty} \widehat{f}(\widehat{x}(\tau), \widehat{y}(\tau)) d \tau<+\infty$.
Since $\widehat{f}(\widehat{x}(\cdot) \cdot \hat{y}(\cdot))$ is uniformly continuous, it follows from Barbalat's lemma that $\dot{z}(t) \longrightarrow 0$ as $t \longrightarrow \infty$ so that $z(t)$ cannot have a limit oscillation in any of its components. So, a stable limit cycle cannot surround the endemic equilibrium point. Therefore, $z(t) \longrightarrow z_{\text {end }}$ as $t \longrightarrow \infty$ for any set of initial conditions satisfying condition (5). Property (ii) has been proved.

Remarks 28. Note that the equilibrium points do not depend on the delays but only on the delay-free and delayed matrices. However, the reproduction numbers are dependent, in general, on each particular set of delays although the threshold $\bar{R}_{0}$ is independent of the delays.

(2) Note also that Theorem 27 concludes the stability (respectively, instability) of the disease-free equilibrium point for each set of delays if $\bar{R}_{0}<1$ (respectively, $\bar{R}_{0}>1$ ). However, it does not conclude that, for a particular set of delays, the endemic equilibrium point is unattainable/ unstable if $\bar{R}_{0}<1$.

(3) From Remark 21, Theorem 13, Corollaries 18 and 19, it follows that $R_{00}$ and $R_{00}$ are the reproduction numbers for the auxiliary delay-free systems AILDFS and AILDDFS, respectively, and also lower bounds of the reproduction number for the whole set of delays. They also guarantee global asymptotic stability independent of the delays towards the disease-free equilibrium under sufficiency-type conditions of tolerance to the delayed dynamics given, respectively, by (44) and (40).

\section{Appendix}

\section{A. Some Technical Results on Positivity and Stability of Matrices and Meztler and $M$-Matrices}

Some technical results on positivity and stability of matrices and their perturbed counterparts are given. Those results are then used concerning the stability of the linearized system around the disease-free equilibrium point.

Theorem A.1 (see [14, 15]). Assume that $G \in \mathbf{R}^{n \times n}$. Then, the following properties hold.

(i) $G$ is an M-matrix if and only if $(-G) \in M_{E}^{n \times n}$.

(ii) $G$ is nonsingular with $G^{-1}>0$ if and only if $G$ is an M-matrix.

(iii) $G$ is nonsingular with $G^{-1}>0$ if and only if $(-G) \in$ $M_{E}^{n \times n}$ is a stability matrix.

(iv) There exists $G_{B}\left(\in \mathbf{R}^{n \times n}\right)>0$ with maximal eigenvalue $r$ such that $G=c I_{n}-G_{B}$, where $c \geq r$.

Theorem A.2. Assume that $G \in \mathbf{R}^{n \times n}$. Then, the following properties hold.

(i) Assume that $G$ is decomposed as $G=G_{1}+G_{2}$ with $G_{1}$ being nonsingular and $G_{2}=G-G_{1}$. Then, $G$ is nonsingular if $\left\|G_{2}\right\|<\varepsilon$ for some sufficiently small (in general, normdependent) $\varepsilon \in \mathbf{R}_{+}$.

(ii) Assume that $G \in \mathbf{R}^{n \times n}$ is an M-matrix that is decomposed as $G=G_{1}+G_{2}$ with $G_{1}$ being an $M$-matrix with 
$G_{1}^{-1}>0$ and $G_{2}=G-G_{1} \prec 0$. Then, $G \in \mathbf{R}^{n \times n}$ is a nonsingular antistable matrix with $G^{-1}>0$ if and only if $\rho\left(G_{2} G_{1}^{-1}\right)<1$. Equivalently, $(-G) \in M_{E}^{n \times n}$, while it is a stability matrix if and only if $\rho\left(G_{2} G_{1}^{-1}\right)<1$.

Proof. Since $\left(G_{1}+G_{2}\right)$ is a regular splitting of $G$ such that $G_{1}$ is nonsingular, one has from Banach's Perturbation Lemma [25] that

$$
G=G_{1}+G_{2}=G_{1}\left(I_{n}+G_{1}^{-1} G_{2}\right)
$$

Thus, $G^{-1}$ exists and

$$
\begin{aligned}
\left\|G^{-1}\right\| & =\left\|\left(G_{1}+G_{2}\right)^{-1}\right\|=\left\|\left(I_{n}+G_{1}^{-1} G_{2}\right)^{-1} G_{1}^{-1}\right\| \\
& \leq\left\|G_{1}^{-1}\right\|\left\|\left(I_{n}+G_{1}^{-1} G_{2}\right)^{-1}\right\| \leq \frac{\left\|G_{d}^{-1}\right\|}{1-\left\|G_{1}^{-1} G_{2}\right\|}
\end{aligned}
$$

for any matrix norm $\|\cdot\|$, provided that $\left\|G_{1}^{-1} G_{2}\right\|<1$, that is, if $\left\|G_{2}\right\|<\varepsilon=1 /\left\|G_{1}^{-1}\right\|=K\left(G_{1}\right) /\left\|G_{1}\right\|$, where $K\left(G_{1}\right)=$ $\left\|G_{1}\right\|\left\|G_{1}^{-1}\right\|$ is the condition number of $G_{1}$ with respect to the matrix norm \| \|. Property (i) has been proved.

To prove Property (ii), note that $\left(G_{1}+G_{2}\right)$ is a regular splitting of the $M$-matrix $G$ since $G_{1}$ is a nonsingular $M$ matrix with $G_{1}^{-1}>0$. Then, $\left(-G_{1}\right) \in M_{E}^{n \times n}$ is a stability matrix (Theorem A.1 (iii)) and $G_{1}$ is anti-stable. Thus, $G_{a 2}=-G_{2}>0$ and $G_{1}^{-1} G_{a 2}>0$ and it follows that

$$
G=G_{1}+G_{2}=G_{1}-G_{a 2}=G_{1}\left(I_{n}-G_{1}^{-1} G_{a 2}\right)
$$

and $G^{-1}$ exists and it is given by $G^{-1}=\left(I_{n}-G_{1}^{-1} G_{a 2}\right)^{-1} G_{1}^{-1}$. Since $\rho\left(G_{1}^{-1} G_{a 2}\right)=\rho\left(G_{1}^{-1} G_{2}\right)<1,\left(I_{n}-G_{1}^{-1} G_{a 2}\right)^{-1}=$ $\sum_{n=0}^{\infty}\left(G_{1}^{-1} G_{a 2}\right)^{n}>0$ from Von Neumann's series expansion and since $\left(G_{1}^{-1} G_{a 2}\right)^{n}>0$ for all $n \geq 0$ and $G_{1}^{-1}>0$, it follows that $G^{-1}=\left(\sum_{n=0}^{\infty}\left(G_{1}^{-1} G_{a 2}\right)^{n}\right) G_{1}^{-1}>0$.

On the other hand, since $G_{a 2}>0$ and $G_{1}^{-1}>0$ both have a simple, in general, nonstrictly dominant Perron root. But the inverse of the Perron root of $G_{1}$ is also a real positive eigenvalue of $G_{1}^{-1}$ with the minimum value of all its eigenvalues. Therefore, since $G_{1}$ and $G_{1}^{-1}$ are both antistable and the eigenvalues of a matrix are continuous function with respect to all its entries, one concludes that the condition $\rho\left(G_{1}^{-1} G_{2}\right)<1$ guarantees that $G^{-1}>0$ and $G$ are both antistable and then $(-G)$ is a stability Metzler matrix. The above condition can also be expressed as $\rho\left(G_{2} G_{1}^{-1}\right)<1$ since the order of the matrix product does not affect the spectral radius since the eigenvalues of a product of two matrices do not depend on the product order [25]. The sufficient part has been proved. The necessity of $\rho\left(G_{2} G_{1}^{-1}\right)<1$ for $G$ to be an antistable $M$-matrix follows since if $\rho\left(G_{2} G_{1}^{-1}\right)=1$ then, from the continuity of all the eigenvalues with respect to the matrix entries, one of the eigenvalues of $G$ crosses the imaginary axis of the complex plane. It has been proved that $G \in \mathbf{R}^{n \times n}$ is a nonsingular antistable matrix with $G^{-1}>0$ if and only if $\rho\left(G_{2} G_{1}^{-1}\right)<1$. Equivalently, $(-G) \in M_{E}^{n \times n}$, while it is a stability matrix, if and only if $\rho\left(G_{2} G_{1}^{-1}\right)<1$.

\section{B. Two Technical Results Which Guarantee the Uniqueness of the Equilibrium Points}

Theorem B.1. The following properties hold.

(i) Let $g: \mathbf{R}^{n+m} \longrightarrow \mathbf{R}^{m}$ be a continuously differentiable function such that $g\left(0, y_{0}\right)=0$ for $0 \in \mathbf{R}^{n}$ and some $y_{0} \epsilon$ $\mathbf{R}^{m}$. If the Jacobian matrix $J_{g, y}\left(0, y_{0}\right)=\left(\left(\partial g_{i} / \partial y_{j}\right)\left(0, y_{0}\right)\right)$ is nonsingular then the disease-free equilibrium point $z_{d f}=$ $\left(0^{T}, y_{d f}^{T}\right)^{T}, y_{d f}=y_{0}$, is unique.

(ii) Let $g: \mathbf{R}^{n+m} \longrightarrow \mathbf{R}^{m}$ be continuous with $g\left(0, y_{0}\right)=0$ for $0 \in \mathbf{R}^{n}$ and some $y_{0} \in \mathbf{R}^{m}$. If there exist open neighborhoods $A \subset \mathbf{R}^{n}$ of $0 \in \mathbf{R}^{n}$ and $B \subset \mathbf{R}^{m}$ of $y_{0}$, such that for all $x \in A$, $g(x,):. A \longrightarrow \mathbf{R}^{m}$ is locally one-to-one then the disease-free equilibrium point $z_{d f}=\left(0^{T}, y_{d f}^{T}\right)^{T}, y_{d f}=y_{0}$, is unique.

Proof. Let $z_{d f}=\left(0^{T}, y_{d f}^{T}\right)^{T}$, for $y_{d f}=y_{0} \in \mathbf{R}^{m}, y_{d f}=$ $y_{01}\left(\neq y_{0}\right) \in \mathbf{R}^{m}$ be two distinct disease-free equilibrium points and $g: \mathbf{R}^{n+m} \longrightarrow \mathbf{R}^{m}$ a continuously differentiable function such that $g\left(0, y_{0}\right)=0$ for $0 \in \mathbf{R}^{n}$ and some $y_{0} \in$ $\mathbf{R}^{m}$. If the Jacobian matrix $J_{g, y}\left(0, y_{0}\right)=\left(\left(\partial g_{i} / \partial y_{j}\right)\left(0, y_{0}\right)\right)$ is nonsingular then, from the implicit function theorem, there exists an open set $U$ of $\mathbf{R}^{n}$ with $0 \in U$ such that there exists a unique continuously differentiable function $h: U \subset \mathbf{R}^{n} \longrightarrow$ $\mathbf{R}^{m}$ such that $y_{0}=h(0), f(x, g(x))=0 ; \forall x \in U$, and $\left(\partial h / \partial x_{j}\right)(x)=-\left[J_{g, y}(x, h(x))\right]^{-1}\left[\left(\partial g / \partial x_{j}\right)(x, h(x))\right] ; \forall x \in U$. Now, take a similar reasoning for $y_{d f}=y_{01}$, again assuming that $J_{g, y}\left(0, y_{01}\right)=\left(\left(\partial g_{i} / \partial y_{j}\right)\left(0, y_{01}\right)\right)$ is nonsingular, what concludes that $y_{01}=h_{1}(0)$ with $h_{1}: U_{1} \longrightarrow R^{m}$ but since both $h: U \longrightarrow R^{m}$ and $h_{1}: U_{1} \longrightarrow R^{m}$ are unique in $U$ and $U_{1}$ which both are open and contain $0 \in \mathbf{R}^{n}$ so that they have a nonempty intersection, it follows that $h \equiv h_{1}$ in $U \cap U_{1}$ so that $y_{01}=h_{1}(0) \neq y_{0}=h(0)$ is a contradiction. Therefore, $z_{d f}=\left(0^{T}, y_{d f}^{T}\right)^{T}$ is unique. Property (i) has been proved. Now, let again $z_{d f}=\left(0^{T}, y_{d f}^{T}\right)^{T}$ be a disease-free equilibrium point with $y_{d f}=y_{0}$. The proof of Property (ii) is supported by the implicit function theorem for continuous (nonnecessarily continuously differentiable) functions. If there exist open neighborhoods $A \subset \mathbf{R}^{n}$ of $0 \in \mathbf{R}^{n}$ and $B \subset \mathbf{R}^{m}$ of $y_{0} \in B$, such that for all $x \in A$, $g(x, \cdot): A \longrightarrow \mathbf{R}^{m}$ is locally one-to-one, then there exist open neighborhoods $A_{0} \in \mathbf{R}^{n}$ of $0 \in \mathbf{R}^{n}$, and $B_{0} \in \mathbf{R}^{m}$ of $y_{0} \in \mathbf{R}^{m}$ such that for all $x \in A_{0}, y=h(x) \in B_{0} \subset \mathbf{R}^{m}$ is the unique local solution of $g(x, y)=0$, defined by the pairs $(x, y=h(x)) \in A_{0} \times B_{0}$, where $h$ is a continuous function from $A_{0}$ into $B_{0}$. Since any disease-free equilibrium point is of the form $z_{d f}=\left(0^{T}, y_{d f}^{T}=h^{T}(0)\right)^{T}$ and $h$ is a continuous function from $A_{0}$, which contains $0 \in \mathbf{R}^{n}$, into $B_{0}$, such an equilibrium point is unique; that is, two solutions $\left(0^{T}, y_{0}^{T}\right)^{T}$ and $z_{d f}=\left(0^{T}, y_{1}^{T}\right)^{T}$ with $y_{0}, y_{1}\left(\neq y_{0}\right)$ cannot exist. Property (ii) has been proved.

Theorem B.2. Assume that all the entries of the Jacobian matrix around any equilibrium point are additive functions and that all the equilibrium points have the same associate transition matrix $\left(-V_{0}\right)$. Let $z_{0}=\left(x_{0}^{T}, y_{0}^{T}\right)^{T}=\left(x_{0}^{T}, h^{T}\left(x_{0}\right)\right)^{T}$ be an endemic equilibrium point. Then, the following properties hold. 
(i) Let $z_{0}$ be reachable. Then, it is the unique endemic reachable equilibrium point if and only if

$$
\begin{aligned}
& {\left[\forall \Delta x_{0}(\neq 0) \in \mathbf{R}^{n}, \forall \Delta y_{0} \in \mathbf{R}^{m}:\left(\Delta x_{0}>-x_{0}\right)\right.} \\
& \quad \wedge\left(h\left(\Delta x_{0}\right)>-y_{0}\right) \wedge\left(f\left(x_{0}+\Delta x_{0}, y_{0}+\Delta y_{0}\right)\right. \\
& \quad \prec 0) \wedge\left(\operatorname { r a n k } \left[F\left(h\left(x_{0}+\Delta x_{0}\right)-h\left(\Delta x_{0}\right)\right)\right.\right. \\
& \left.\left.\quad+F\left(h\left(\Delta x_{0}\right)\right)-2 V_{0}, 2 f\left(\Delta x_{0}, h\left(\Delta x_{0}\right)\right)\right]\right) \\
& \quad=\operatorname{rank}\left[F\left(h\left(x_{0}+\Delta x_{0}\right)-h\left(\Delta x_{0}\right)\right)+F\left(h\left(\Delta x_{0}\right)\right)\right. \\
& \left.\left.\quad-2 V_{0}\right]\right] \Longrightarrow\left[h\left(x_{0}\right) \neq h\left(x_{0}+\Delta x_{0}\right)-h\left(\Delta x_{0}\right)\right]
\end{aligned}
$$

(ii) Let $z_{0}$ be either reachable or unattainable. Then, it is the unique endemic equilibrium point if and only if

$$
\begin{aligned}
& {\left[\forall \Delta x_{0}(\neq 0) \in \mathbf{R}^{n}, \forall \Delta y_{0} \in \mathbf{R}^{m}:\right.} \\
& \quad\left(\operatorname { r a n k } \left[F\left(h\left(x_{0}+\Delta x_{0}\right)-h\left(\Delta x_{0}\right)\right)+F\left(h\left(\Delta x_{0}\right)\right)\right.\right. \\
& \left.\left.\quad-2 V_{0}, 2 f\left(\Delta x_{0}, h\left(\Delta x_{0}\right)\right)\right]\right) \\
& \quad=\operatorname{rank}\left[F\left(h\left(x_{0}+\Delta x_{0}\right)-h\left(\Delta x_{0}\right)\right)\right. \\
& \left.\left.\quad+F\left(h\left(\Delta x_{0}\right)\right)-2 V_{0}\right]\right] \Longrightarrow\left[h\left(x_{0}\right) \neq h\left(x_{0}+\Delta x_{0}\right)\right. \\
& \left.\quad-h\left(\Delta x_{0}\right)\right]
\end{aligned}
$$

Proof. Assume that there exist two distinct equilibrium points with infective and noninfective variables $x_{(\cdot)}$ and $y_{(\cdot)}$, namely, $z_{0}=\left(x_{0}^{T}, y_{0}^{T}\right)^{T}$ and $z_{1}=\left(x_{1}^{T}, y_{1}^{T}\right)^{T}=z_{0}+\Delta z_{0}$ so that

$$
\begin{aligned}
J_{g, z}\left(z_{1}\right) z_{1} & =J_{g, z}\left(z_{0}+\Delta z_{0}\right)\left(z_{0}+\Delta z_{0}\right) \\
& =J_{g, z}\left(z_{0}\right) z_{0}=0
\end{aligned}
$$

Since all the entries of the Jacobian matrices are additive, one gets

$$
\begin{aligned}
& J_{g, z}\left(z_{1}\right) z_{1}-J_{g, z}\left(z_{0}\right) z_{0} \\
& \quad=J_{g, z}\left(z_{0}\right) \Delta z_{0}+J_{g, z}\left(\Delta z_{0}\right) z_{0}+J_{g, z}\left(\Delta z_{0}\right) \Delta z_{0} \\
& \quad=0
\end{aligned}
$$

then

$$
J_{g, z}\left(z_{0}\right) \Delta z_{0}+J_{g, z}\left(\Delta z_{0}\right) \Delta z_{0}=-J_{g, z}\left(\Delta z_{0}\right) z_{0} .
$$

Again, using the additive property assumption on the Jacobian matrix, one has that the infective and noninfective compartments satisfy the constraints:

$$
\begin{aligned}
& \left(F\left(y_{0}\right)-V_{0}\right) \Delta x_{0}-f\left(x_{0}, y_{0}\right)+\left(F\left(\Delta y_{0}\right)-V_{0}\right) \Delta x_{0} \\
& \quad-f\left(\Delta x_{0}, \Delta y_{0}\right)=-\left(F\left(\Delta y_{0}\right)-V_{0}\right) x_{0} \\
& \quad+f\left(\Delta x_{0}, \Delta y_{0}\right) \\
& y_{0}=h\left(x_{0}\right) \\
& y_{1}=h\left(x_{1}\right)=h\left(x_{0}+\Delta x_{0}\right)=y_{0}+h\left(\Delta x_{0}\right)=y_{0} \\
& \quad+\Delta y_{0}
\end{aligned}
$$

where $\Delta x_{0}=x_{1}-x_{0}$ and $\Delta y_{0}=y_{1}-y_{0}$. If the second two constraints are replaced into the first equation, one gets that, from Rouché-Froebenius theorem of Linear Algebra, such an algebraic equation is solvable (being either compatible determinate with one solution or indeterminate with infinitely many ones) in $\Delta x_{0} \neq 0$ if and only if

$$
\begin{aligned}
\operatorname{rank} & {\left[F\left(h\left(x_{0}+\Delta x_{0}\right)-h\left(\Delta x_{0}\right)\right)+F\left(h\left(\Delta x_{0}\right)\right)\right.} \\
& \left.-2 V_{0}, 2 f\left(\Delta x_{0}, h\left(\Delta x_{0}\right)\right)\right] \\
& =\operatorname{rank}\left[F\left(h\left(x_{0}+\Delta x_{0}\right)-h\left(\Delta x_{0}\right)\right)\right. \\
& \left.\left.+F\left(h\left(\Delta x_{0}\right)\right)-2 V_{0}\right]\right]
\end{aligned}
$$

This implies the existence of at least another endemic equilibrium point, which is not coincident with the disease-free one since $x_{1}=\Delta x_{0} \neq 0$ if $h\left(y_{1}\right)=h\left(x_{0}+\Delta x_{0}\right)=h\left(x_{0}\right)+$ $h\left(\Delta x_{0}\right)=y_{0}+h\left(\Delta x_{0}\right)$. Such a point does not exist if either rank condition fails (i.e., the algebraic system is incompatible) or if it holds but $h\left(x_{0}\right) \neq h\left(x_{0}+\Delta x_{0}\right)-h\left(\Delta x_{0}\right)$. There are additional conditions on positivity needed for the point to be reachable in the case that it exists. This leads directly to the two claimed properties.

\section{Data Availability}

Previously reported data used to support this study are cited at relevant places within the text referred to the references in the manuscript.

\section{Conflicts of Interest}

The authors declare that there is no conflict of interests regarding the publication of this paper.

\section{Acknowledgments}

The authors are grateful to the Spanish Government for Grants DPI2015-64766-R, RTI2018-094336-B-I00, and DPI2016-77271-R (MINECO/FEDER, UE).

\section{References}

[1] Z. Shuai and P. Van Den Driessche, "Global stability of infectious disease models using Lyapunov functions," SIAM Journal on Applied Mathematics, vol. 73, no. 4, pp. 1513-1532, 2013.

[2] P. van den Driessche and J. Watmough, "Reproduction numbers and sub-threshold endemic equilibria for compartmental models of disease transmission," Mathematical Biosciences, vol. 180, pp. 29-48, 2002.

[3] P. Van Den Driessche and J. Watmough, "Further notes on the reproduction number in mathematical epidemiology," in Lecture Notes in Mathematics, F. Brauer, P. Van den Driessche, and J. Wu, Eds., pp. 159-178, Springer, Berlin, Germany, 2008.

[4] O. Diekmann, J. A. P. Heesterbeek, and M. G. Roberts, "The construction of next-generation matrices for compartmental epidemic models," Journal of the Royal Society Interface, vol. 7, no. 47, pp. 873-885, 2010. 
[5] B. Ridenhour, J. M. Kowalik, and D. K. Say, "Unraveling: Considerations for public health applications," Pan American Journal of Public Health, vol. 38, no. 2, pp. 167-176, 2015.

[6] M. De la Sen, A. Ibeas, S. Alonso-Quesada, and R. Nistal, "On a new epidemic model with asymptomatic and dead-infective subpopulations with feedback controls useful for ebola disease," Discrete Dynamics in Nature and Society, vol. 2017, Article ID 4232971, 22 pages, 2017.

[7] M. De la Sen, R. P. Agarwal, R. Nistal, S. Alonso-Quesada, and A. Ibeas, "A switched multicontroller for an SEIADR epidemic model with monitored equilibrium points and supervised transients and vaccination costs," Advances in Difference Equations, vol. 2018, no. 1, article number 390, 2018.

[8] S. Alonso-Quesada, M. De la Sen, and A. Ibeas, "On the discretization and control of an SEIR epidemic model with a periodic impulsive vaccination," Communications in Nonlinear Science and Numerical Simulation, vol. 42, pp. 247-274, 2017.

[9] D. Kiouach and Y. Sabbar, "Stability and threshold of a stochastic SIRS epidemic model with vertical transmission and transfer from infectious to susceptible individuals," Discrete Dynamics in Nature and Society, vol. 2018, Article ID 7570296, 13 pages, 2018.

[10] D. Kiouach and L. Boulaasair, "Stationary distribution and dynamic behaviour of a stochastic SIVR epidemic model with imperfect vaccine," Journal of Applied Mathematics, vol. 2018, pp. 1-11, 2018.

[11] W. Xia, S. Kundu, and S. Maitra, "Dynamics of a delayed SEIQ epidemic model," Advances in Difference Equations, vol. 2018, no. 1, p. 336, 2018.

[12] N. Shamsi G., S. Ali Torabi, and G. H. Shakouri, "An option contract for vaccine procurement using the SIR epidemic model," European Journal of Operational Research, vol. 267, no. 3, pp. 1122-1140, 2018.

[13] M. De la Sen and S. Alonso-Quesada, "Control issues for the Beverton-Holt equation in ecology by locally monitoring the environment carrying capacity: non-adaptive and adaptive cases," Applied Mathematics and Computation, vol. 215, no. 7, pp. 2616-2633, 2009.

[14] T. Kaczorek, Positive $1 D$ and 2D Systems, Springer-Verlag, Berlin, Germany, 2002.

[15] G. Giorgio and C. Zuccotti, "Metzlerian and generalized metzlerian matrices: some properties and economic applications," Journal of Mathematics Research, vol. 7, no. 2, pp. 42-55, 2015.

[16] C.-K. Li and H. Schneider, "Applications of perron-frobenius theory to population dynamics," Journal of Mathematical Biology, vol. 44, no. 5, pp. 450-462, 2002.

[17] L. Edelstein-Keshet, Mathematical Models in Biology, vol. 46 of Classics in Applied Mathematics, SIAM, Philadelphia, Pa, USA, 2005.

[18] D. S. Niculescu, Delay Effects on Stability: A Robust Control Approach, Springer, London, UK, 2001.

[19] M. de la Sen, "On positivity of singular regular linear timedelay time-invariant systems subject to multiple internal and external incommensurate point delays," Applied Mathematics and Computation, vol. 190, no. 1, pp. 382-401, 2007.

[20] M. de la Sen, "Quadratic stability and stabilization of switched dynamic systems with uncommensurate internal point delays," Applied Mathematics and Computation, vol. 185, no. 1, pp. 508526, 2007.

[21] Y. Zhang, Q. Zhang, T. Tanaka, and X.-G. Yan, "Positivity of continuous-time descriptor systems with time delays," IEEE Transactions on Automatic Control, vol. 59, no. 11, pp. 30933097, 2014.
[22] F. Sun, L. Zhou, Q. Zhang, and Y. Shen, "Stability bound analysis and synthesis for singularly perturbed systems with time-varying delay," Mathematical Problems in Engineering, vol. 2013, Article ID 517258, 8 pages, 2013.

[23] G. Rajchakit, T. Rojsiraphisal, and M. Rajchakit, "Robust stability and stabilization of uncertain switched discrete-time systems," Advances in Difference Equations, vol. 2012, no. 1, article 134, 15 pages, 2012.

[24] S. Sastry and M. Bodson, Adaptive Control Stability, Convergence and Robustness, Prentice-Hall, Englewood Cliffs, NJ, USA, 1989.

[25] J. M. Ortega, Numerical Analysis, Academic Press, New York, NU, USA, 1972.

[26] F. Kittaneh, "Spectral radius inequalities for Hilbert space operators," Proceedings of the American Mathematical Society, vol. 134, no. 2, pp. 385-390, 2006.

[27] O. Diekmann, H. Heesterbeek, and H. Metz, "The legacy of Kermack and McKendrick," in Epidemic Models: Their Structure and Relation to Data, pp. 95-115, Cambridge University Press, New York, NY, USA, 1995.

[28] H. Gorecki, S. Fuksa, P. Grabowsi, and A. Korytowski, Analysis and Synthesis of Time Delay Systems, John Wiley and Sons, Chichester, UK, 1989. 


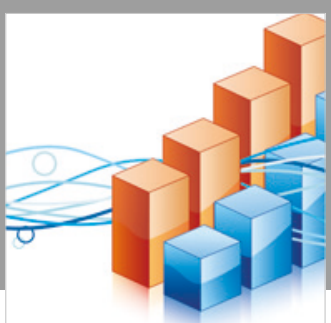

Advances in

Operations Research

\section{-n-m}
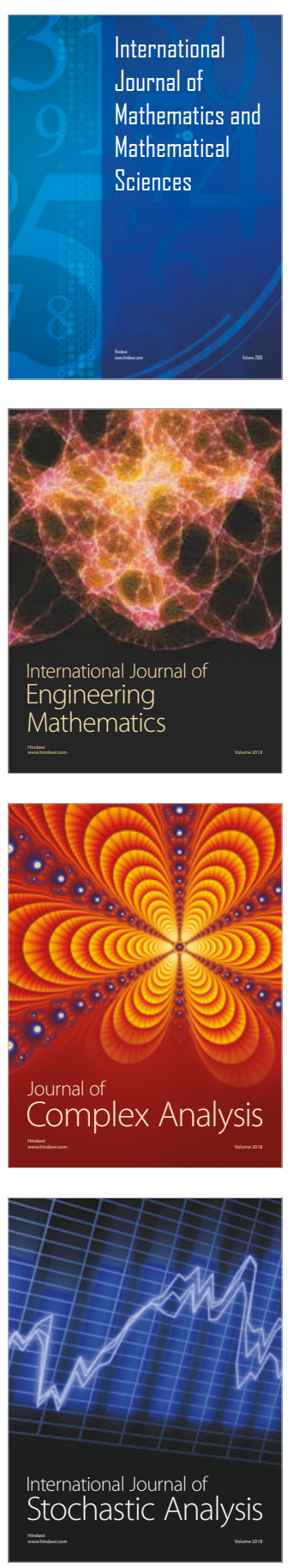
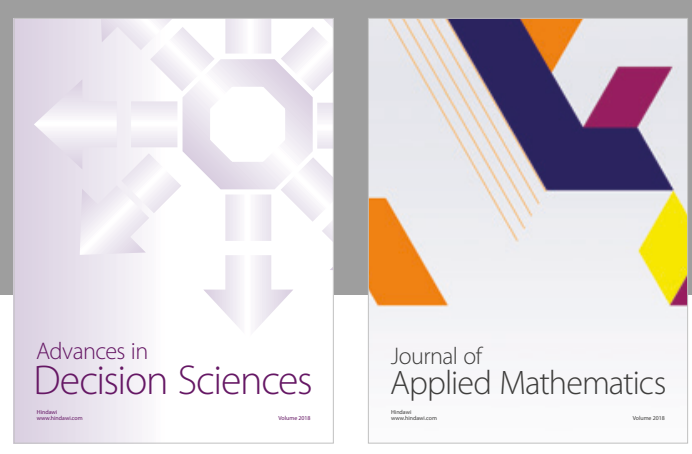

Journal of

Applied Mathematics
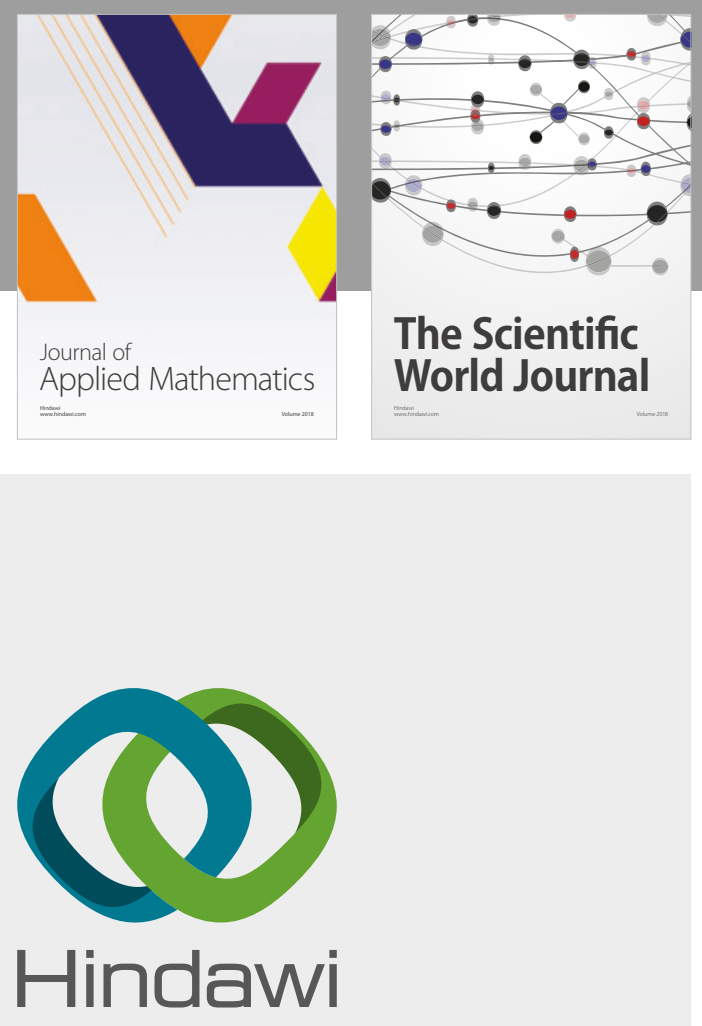

Submit your manuscripts at

www.hindawi.com

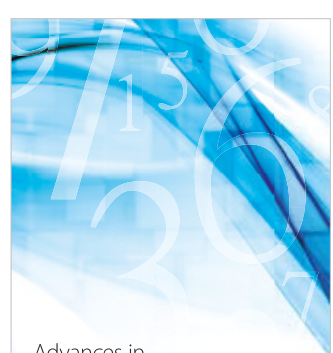

Advances in
Numerical Analysis
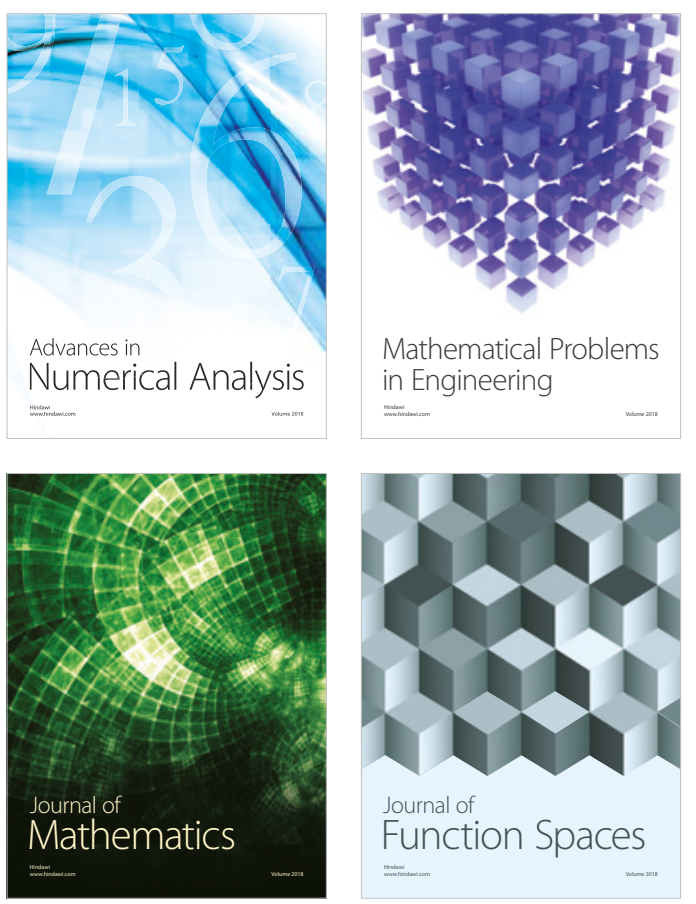

Mathematical Problems in Engineering

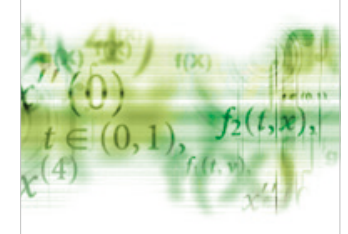

International Journal of

Differential Equations

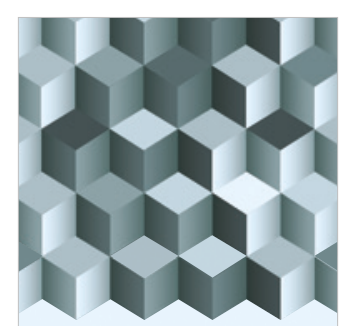

Journal of

Function Spaces
The Scientific

World Journal

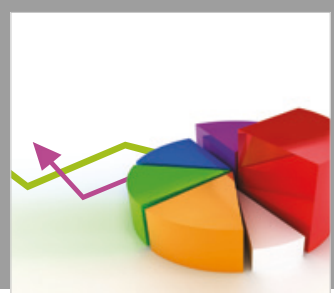

Journal of

Probability and Statistics
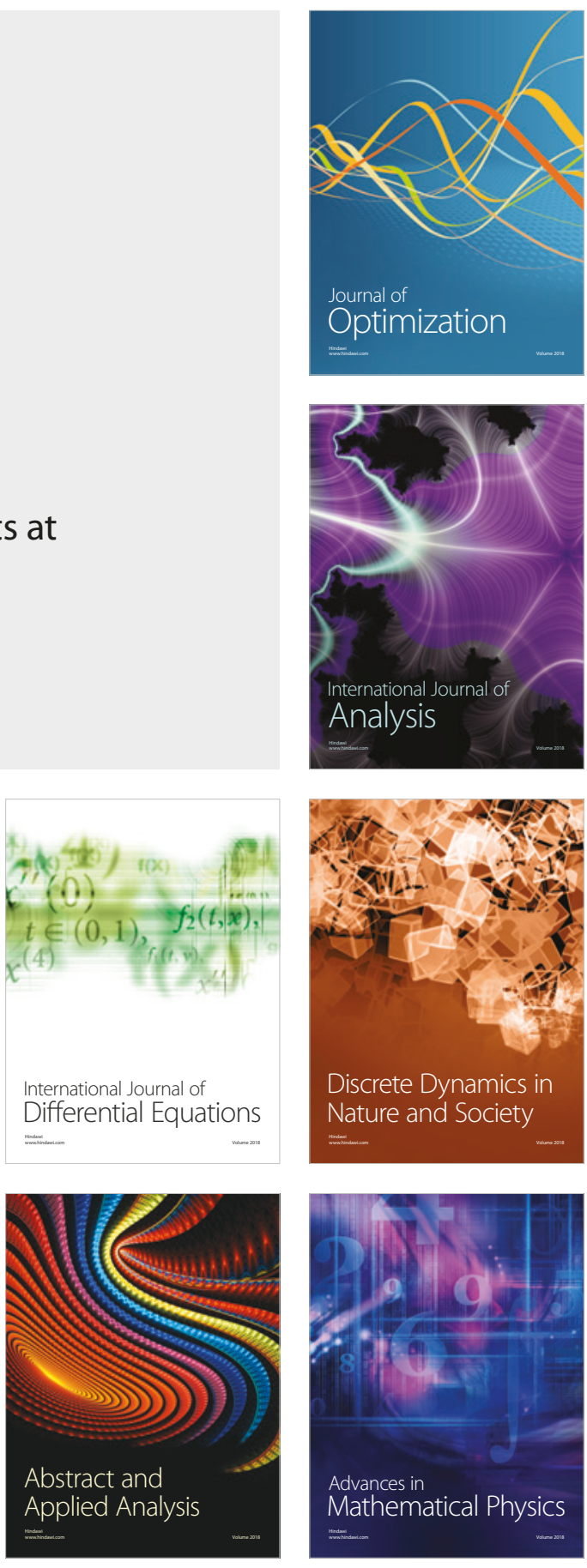